MATHEMATICS OF COMPUTATION

Volume 73, Number 247, Pages 1261-1296

S 0025-5718(04)01638-2

Article electronically published on March 3, 2004

\title{
EXISTENCE AND ASYMPTOTIC STABILITY OF RELAXATION DISCRETE SHOCK PROFILES
}

\author{
MAO YE
}

\begin{abstract}
In this paper we study the asymptotic nonlinear stability of discrete shocks of the relaxing scheme for approximating the general system of nonlinear hyperbolic conservation laws. The existence of discrete shocks is established by suitable manifold construction, and it is shown that weak single discrete shocks for such a scheme are nonlinearly stable in $L^{2}$, provided that the sums of the initial perturbations equal zero. These results should shed light on the convergence of the numerical solution constructed by the relaxing scheme for the single shock solution of the system of hyperbolic conservation laws. These results are proved by using both a weighted norm estimate and a characteristic energy method based on the internal structures of the discrete shocks.
\end{abstract}

\section{INTRODUCTION}

We investigate the asymptotic stability of the numerical approximation of the following Riemann problem for the general system of nonlinear conservation laws

$$
u_{t}+F(u)_{x}=0, u(x, 0)=u_{0}(x)= \begin{cases}u_{-}, & x<0 \\ u_{+}, & x>0\end{cases}
$$

where $u=u(x, t) \in \mathbb{R}^{m}, F$ is a smooth nonlinear mapping from $\mathbb{R}^{m}$ to $\mathbb{R}^{m}$, and $u_{ \pm}$are two constant vectors in $\mathbb{R}^{m}$. We assume that the system (1.1) is strictly hyperbolic in the sense that at each state $u \in \mathbb{R}^{m}$ the Jacobian $\nabla F(u)$ has $m$ real and distinct eigenvalues

$$
\lambda_{1}(u)<\lambda_{2}(u)<\cdots<\lambda_{m}(u)
$$

with corresponding left and right eigenvectors $l_{\mu}$ and $r_{\mu}$, respectively, and the characteristic field is either genuinely nonlinear or linearly degenerate in the sense of $\operatorname{Lax}$ [12; i.e., for $\mu=1, \ldots, m$, the eigenvector $r_{\mu}$ satisfies $\nabla \lambda_{\mu} \cdot r_{\mu} \equiv 1$ or $\nabla \lambda_{\mu} \cdot r_{\mu} \equiv 0$. We normalize the eigenvectors so that $l_{\mu} r_{\kappa}=\delta_{\mu \kappa}$ and we denote the $m \times m$ matrices $L(u), R(u)$ and $\Lambda(u)$ by

$$
\begin{gathered}
L(u)=\left(l_{1}(u)^{T}, \ldots, l_{m}(u)^{T}\right)^{T}, R(u)=\left(r_{1}(u), \ldots, r_{m}(u)\right), \\
\Lambda(u)=\operatorname{diag}\left(\lambda_{1}(u), \ldots, \lambda_{m}(u)\right) .
\end{gathered}
$$

Received by the editor September 3, 2002.

2000 Mathematics Subject Classification. Primary 65M12; Secondary 35L65.

Key words and phrases. Relaxing scheme, hyperbolic systems of conservation laws, discrete shock profiles, nonlinear stability.

This work was supported by the Youth Science and Technology Foundation, UESTC YF020801. 
Lax [12] showed that the Riemann problem (1.1) has a solution consisting of at most $(m+1)$ constant states separated by shock waves, centered rarefaction waves, and contact discontinuities, provided that the shock strength $\left|u_{+}-u_{-}\right|$is suitably small.

Assume that the Riemann problem (1.1) has a $k$-shock wave solution

$$
u(x, t)= \begin{cases}u_{-}, & x<s t \\ u_{+}, & x>s t\end{cases}
$$

corresponding to the $k$-th genuinely nonlinear field. Here the constant states $u_{ \pm}$ and the shock speed $s$ satisfy the Rankine-Hugoniot condition

$$
F\left(u_{-}\right)-F\left(u_{+}\right)=s\left(u_{-}-u_{+}\right)
$$

and the Lax entropy condition

$$
\lambda_{k}\left(u_{+}\right)<s<\lambda_{k}\left(u_{-}\right) .
$$

We approximate (1.1) with the relaxing scheme proposed by Jin and Xin [9],

$$
\begin{gathered}
u_{j}^{n+1}-u_{j}^{n}+\frac{k}{2 h}\left(v_{j+1}^{n}-v_{j-1}^{n}\right)-\frac{k}{2 h} A^{\frac{1}{2}}\left(u_{j+1}^{n}-2 u_{j}^{n}+u_{j-1}^{n}\right)=0, \\
v_{j}^{n+1}-v_{j}^{n}+\frac{k}{2 h} A\left(u_{j+1}^{n}-u_{j-1}^{n}\right)-\frac{k}{2 h} A^{\frac{1}{2}}\left(v_{j+1}^{n}-2 v_{j}^{n}+v_{j-1}^{n}\right) \\
=\kappa\left(F\left(u_{j}^{n+1}\right)-v_{j}^{n+1}\right) .
\end{gathered}
$$

$\kappa$ is sufficiently large, $u_{j}^{n}$ is the approximation of $u\left(x_{j}, t^{n}\right), x_{j}=j \Delta x$ and $t^{n}=n \Delta t$, $\Delta x$ and $\Delta t$ are the spatial and temporal grid sizes. $\lambda=\frac{k}{h}$ is sufficiently small, which satisfies the Courant-Friedrichs-Lwey (CFL) condition

$$
\lambda \sup _{\mu}\left|\lambda_{\mu}(u)\right| \leq 1
$$

$A=a I$ and $A^{\frac{1}{2}}=a^{\frac{1}{2}} I$. The relaxing scheme (1.2) satisfies the strict subcharacteristic condition which implies stability in some sense.

A relaxation discrete shock profile connecting $\left(u_{-}, F\left(u_{-}\right)\right)$and $\left(u_{+}, F\left(u_{+}\right)\right)$is a special solution of the difference equation (1.2) in the form

$$
\begin{gathered}
u_{x-\eta}-u_{x}+\frac{k}{2 h}\left(v_{x+1}-v_{x-1}\right)-\frac{k}{2 h} A^{\frac{1}{2}}\left(u_{x+1}-2 u_{x}+u_{x-1}\right)=0 \\
v_{x-\eta}-v_{x}+\frac{k}{2 h} A\left(u_{x+1}-u_{x-1}\right)-\frac{k}{2 h} A^{\frac{1}{2}}\left(v_{x+1}-2 v_{x}+v_{x-1}\right) \\
=\kappa\left(F\left(u_{x-\eta}\right)-v_{x-\eta}\right)
\end{gathered}
$$

with

$$
\begin{aligned}
\lim _{x \rightarrow+\infty}\left(u_{x}, v_{x}\right) & =\left(u_{+}, F\left(u_{+}\right)\right), \\
\lim _{x \rightarrow-\infty}\left(u_{x}, v_{x}\right) & =\left(u_{-}, F\left(u_{-}\right)\right) .
\end{aligned}
$$

When $\eta$ is a rational number, $x \in\{n \eta+m \mid n, m \in \mathbb{Z}\}$; if $\eta$ is an irrational number, $x \in \mathbb{R}$. Let $u_{-}$and $u_{+}$satisfy the Rankine-Hugoniot condition

$$
F\left(u_{-}\right)-F\left(u_{+}\right)=s\left(u_{-}-u_{+}\right) .
$$

The discrete shock speed $\eta$ is related to $s$ on the condition that $\lambda s=\eta$. In this paper we require that this discrete shock can be observed on the original grid; therefore, we assume that $\eta=p / q$ is rational. Thus, the discrete shock profiles 
which we have studied are the solutions of the difference equations satisfying

$$
\begin{gathered}
\phi_{j}^{n+1}-\phi_{j}^{n}+\frac{k}{2 h}\left(\psi_{j+1}^{n}-\psi_{j-1}^{n}\right)-\frac{k}{2 h} A^{\frac{1}{2}}\left(\phi_{j+1}^{n}-2 \phi_{j}^{n}+\phi_{j-1}^{n}\right)=0, \\
\psi_{j}^{n+1}-\psi_{j}^{n}+\frac{k}{2 h} A\left(\phi_{j+1}^{n}-\phi_{j-1}^{n}\right)-\frac{k}{2 h} A^{\frac{1}{2}}\left(\psi_{j+1}^{n}-2 \psi_{j}^{n}+\psi_{j-1}^{n}\right) \\
=\kappa\left(F\left(\phi_{j}^{n+1}\right)-\psi_{j}^{n+1}\right) \\
\left(\phi_{j}^{n q}, \psi_{j}^{n q}\right)=\left(\phi_{j-n p}^{0}, \psi_{j-n p}^{0}\right), \\
\left(\phi_{j}, \psi_{j}\right) \rightarrow\left(u_{ \pm}, f\left(u_{ \pm}\right)\right), j \rightarrow \pm \infty .
\end{gathered}
$$

The study of the existence and stability of discrete shock profiles is important for understanding the convergence behavior of numerical shock computations. In the study of discrete conservation laws, Jennings [10] showed the stability of discrete shock profiles of general scalar first-order monotone schemes. The existence of discrete shock profiles of high order scalar difference scheme was established in 4, 8. recently. For the study of the system of conservation laws, the existence of discrete shock profiles of finite-difference methods which are accurate to first order for systems of conservation laws was established by Majda and Ralson [21] using the center manifold construction and by Michelson in 22. Liu and Xin [16] 17] studied the nonlinear stability of discrete shock profiles of system of conservation laws. Liu and $\mathrm{Yu}$ 19, 20] studied the case of more general shock speeds. There are several authors who have studied the stability of shock profiles; see [2, 3, 5, 6, 25, 26. In the study of discrete relaxation conservation laws, most works have concentrated on the scalar case, such as Liu, Wang and Yang [13, 14, 15. In this paper, we will concentrate on the case of systems. Stability theorems of discrete shock profiles follow. Their existence will be proved in section 2. Note that we assume that $\lambda$ is suitably small in all the following theorems.

Theorem 1.1. Suppose that (1.1) is a strictly hyperbolic system and that the $k$ characteristic field is genuinely nonlinear. Let $\left(\phi_{j}, \psi_{j}\right)$ be the stationary discrete shock profile of (1.3) connecting $\left(u_{-}, f\left(u_{-}\right)\right)$and $\left(u_{+}, f\left(u_{+}\right)\right)$. Assume further that

$$
\begin{gathered}
\sum_{j=-\infty}^{\infty}\left(u_{j}^{0}-\phi_{j}, v_{j}^{0}-\psi_{j}\right)=0, \\
\epsilon=\left|u_{+}-u_{-}\right| \leq c_{1}
\end{gathered}
$$

and

$$
\sum_{j=-\infty}^{\infty}\left(1+j^{2}\right)\left(\left|u_{j}^{0}-\phi_{j}\right|^{2},\left|v_{j}^{0}-\psi_{j}\right|^{2}\right) \leq c_{2}
$$

for some (suitably small) positive constants $c_{1}$ and $c_{2}$. Then, there exists a unique global solution $\left(u_{j}^{n}, v_{j}^{n}\right)$ to the relaxing scheme with initial data $\left(u_{j}^{0}, v_{j}^{0}\right)$, and it satisfies

$$
\lim _{n \rightarrow+\infty} \sum_{j=-\infty}^{\infty}\left(\left|u_{j}^{n}-\phi_{j}\right|^{2},\left|v_{j}^{n}-\psi_{j}\right|^{2}\right)=0 .
$$

Theorem 1.2. Suppose that (1.1) is a strictly hyperbolic system and that the $k$ characteristic field is genuinely nonlinear. Let $\left(\phi_{j}^{n}, \psi_{j}^{n}\right)$ be the nonstationary discrete shock profile of (1.3) connecting $\left(u_{-}, f\left(u_{-}\right)\right)$and $\left(u_{+}, f\left(u_{+}\right)\right)$. Assume that $\kappa$ is suitably large,

$$
\begin{gathered}
\sum_{j=-\infty}^{\infty}\left(u_{j}^{0}-\phi_{j}^{0}, v_{j}^{0}-\psi_{j}^{0}\right)=0, \\
\epsilon=\left|u_{+}-u_{-}\right| \leq c_{1}
\end{gathered}
$$


and

$$
\sum_{j=-\infty}^{\infty}\left(1+j^{2}\right)\left(\left|u_{j}^{0}-\phi_{j}^{0}\right|^{2},\left|v_{j}^{0}-\psi_{j}^{0}\right|^{2}\right) \leq c_{2}
$$

for some (suitably small) positive constants $c_{1}$ and $c_{2}$. Then there exists a unique global solution $\left(u_{j}^{n}, v_{j}^{n}\right)$ to the relaxing scheme with initial data $\left(u_{j}^{0}, v_{j}^{0}\right)$, and it satisfies

$$
\lim _{n \rightarrow+\infty} \sum_{j=-\infty}^{\infty}\left(\left|u_{j}^{n}-\phi_{j}^{n}\right|^{2},\left|v_{j}^{n}-\psi_{j}^{n}\right|^{2}\right)=0 .
$$

Remark 1.1. For the stationary case, we do not need to assume that $\kappa$ is suitably large. This condition is only imposed in the nonstationary case due to technical reasons when we estimate the nonstationary discrete shock profiles in (4.8). However, this condition also implies that the relaxing scheme (1.2) approximates to the conservation laws (1.1) more closely.

Remark 1.2. (1.5),(1.7),(1.9) and (1.11) imply that the $L^{2}$ norm of initial perturbation is sufficiently small; see [7, 16].

Remark 1.3. Although there are some essential monotone properties in the principal direction, the multistep phenomena result in some difficulties in studying the $L^{1}$ stability, which is kept for the future.

Remark 1.4. Only partial results are obtained, i.e., when $\eta$ is a rational number, and there is no complete understanding if $\eta$ is an arbitrary number. For a diophantine number [19], we need to use the Green function of the relaxing scheme, which is not very clear up to this point.

The outline of this paper is as follows. In Section 2, we study the existence of relaxation discrete shock profiles using the Majda-Ralston [21] theorem. Then, we obtain some good properties of the shock profiles using the theorems of Liu-Xin [16]. In Section 3, we study the $L^{2}$ stability of stationary discrete shock profiles using the suitable weighted functions proposed by Liu-Xin in [17]. In Section 4, we study the $L^{2}$ stability of nonstationary discrete shock profiles using the idea of vertical estimate.

\section{EXISTENCE OF DISCRETE SHOCK PROFILES}

To establish the existence of discrete shock profiles for the relaxation scheme, we will use the center manifold construction proposed by Majda-Ralston [21. Let $T: \mathbb{R}^{N} \rightarrow \mathbb{R}^{N}$ be a smooth map and let $x_{0} \in \mathbb{R}^{N}$ be a fixed point of $T$ such that the following conditions are satisfied.

(1) There are neighborhoods $\mu$ and $\mu^{\prime}$ of $x_{0}$ in $\mathbb{R}^{N}$ such that $T$ maps $\mu$ diffeomorphically onto $\mu^{\prime}$.

(2) $T$ fixes a smooth $m$-dimensional manifold $\Omega$ containing $x_{0}$.

(3) The algebraic eigenspace of $d T\left(x_{0}\right)$ associated with the eigenvalue 1 is the span of the tangent space of $\Omega, \Gamma_{x_{0}}(\Omega)$ and a vector $\chi$ satisfying

$$
d T\left(x_{0}\right) \chi=\chi+r,
$$

where $r \in \Gamma_{x_{0}}(\Omega)$ and $r \neq 0$.

(4) $d T\left(x_{0}\right)$ has no eigenvalues in the set $\{z:|z|=1, z \neq 1\}$. 
(5) Hypotheses (2) and (3) imply that given $\delta^{\prime}>0$, there is a $\delta>0$ such that when $x \in \Omega$ and $\left|x-x_{0}\right|<\delta$, the eigenvalues of $d T(x)$ lying in $|z-1|<\delta^{\prime}$ are 1 with multiplicity $m$ and real $\beta(x)$ with multiplicity 1 where $\beta(x) \in C^{\infty}(\Omega)$, and $\left.\nabla \beta \cdot r\right|_{x_{0}}>0$.

We summarize the center manifold construction for such an operator in the following proposition.

Proposition 2.1 ([21]). Assume that the map T satisfies condition (1)-(5). Then there is an $\epsilon>0$ such that if $x_{-} \in \Omega, \beta\left(x_{-}\right)>1$ and $\left|x_{-}-x_{0}\right|<\epsilon$, then

(1) there is a $C^{1}$ segment $\gamma_{x_{-}}$beginning at $x_{-}$such that $T^{-1} \gamma_{x_{-}} \subset \gamma_{x_{-}}$and $\bigcap_{j} T^{-j} \gamma_{x_{-}}=x_{-}$

(2) for any $x \in \gamma_{x_{-}}, x \neq x_{-}$, the sequence $T^{j} x, j=0,1, \ldots$ converges to $x_{+} \in \Omega$ with $\beta\left(x_{+}\right)<1$,

(3) if the limit of $T^{j} x$ in (2) does not depend on $x$, then $\bigcup_{j=0} T^{j} \gamma_{x_{-}}=\Gamma_{x_{-}}$is a $C^{1}$ curve segment beginning at $x_{-} \in \Omega, \beta\left(x_{-}\right)>1$, and ending at $x_{+} \in \Omega$, $\beta\left(x_{+}\right)<1$.

Remark 2.1. Item (3) is guaranteed here by the Rankine-Hugoniot condition and the Lax entropy condition $\lambda_{k}\left(u_{-}\right)>s>\lambda_{k}\left(u_{+}\right)$, and $u_{+}$is uniquely determined by $u_{-}$. This will be clear in the remainder of this section.

Our strategy is using this proposition to prove the existence of discrete shock profiles of the $u$ component first and then the $v$ component. It follows from equation (1.3) that

$$
\frac{\lambda}{2}\left(v_{x+1}-v_{x-1}\right)=\frac{\lambda}{2} A^{\frac{1}{2}}\left(u_{x+1}-2 u_{x}+u_{x-1}\right)-\left(u_{x-\eta}-u_{x}\right),
$$

so the second equation of (1.3) yields

$$
\begin{gathered}
\frac{\lambda}{2}\left(v_{x+1-\eta}-v_{x-1-\eta}\right)-\frac{\lambda}{2}\left(v_{x+1}-v_{x-1}\right)+\frac{\lambda^{2}}{4} A\left(u_{x+2}-2 u_{x}+u_{x-2}\right) \\
\quad-\frac{\lambda}{2} A^{\frac{1}{2}}\left[\frac{\lambda}{2}\left(v_{x+2}-v_{x}\right)-\lambda\left(v_{x+1}-v_{x-1}\right)+\frac{\lambda}{2}\left(v_{x}-v_{x-2}\right)\right] \\
\quad=\frac{\kappa \lambda}{2}\left(F\left(u_{x+1-\eta}\right)-F\left(u_{x-1-\eta}\right)\right)-\frac{\kappa \lambda}{2}\left(v_{x+1}-\eta v_{x-1-\eta}\right)
\end{gathered}
$$

For simplicity, we only consider $\eta=\frac{1}{q}$ and $q>0$, and the other cases are similar. Some remarks about the case of $s>0$ will also be given later. Substituting (2.2) into (2.3), scaling the step size as $\Delta x / q$, and writing $\tilde{u}_{j}=u(j \Delta x / q)$, we have the following equation,

$$
\begin{aligned}
& {\left[\frac{\lambda}{2} A^{\frac{1}{2}}(1+\kappa)+\frac{\lambda}{2} A^{\frac{1}{2}}\right] \tilde{u}_{j+2 q+1}+\left[\left(1-A^{\frac{1}{2}}\right)(1+\kappa)+1-A^{\frac{1}{2}} \lambda\right] \tilde{u}_{j+q+1}} \\
& +\left[\frac{\lambda}{2} A^{\frac{1}{2}}(1+\kappa)+\frac{\lambda}{2} A^{\frac{1}{2}}\right] \tilde{u}_{j+1}-[1+\kappa] \tilde{u}_{j+q}+\left[2 \lambda A^{\frac{1}{2}}-2 \lambda^{2} A-1\right] \tilde{u}_{j+q+2} \\
& +\left[\lambda^{2} A-\lambda A^{\frac{1}{2}}\right] \tilde{u}_{j+2}-\frac{\lambda}{2} \kappa\left[F\left(\tilde{u}_{j+2 q+1}\right)-F\left(\tilde{u}_{j+1}\right)\right]=\left[\lambda A^{\frac{1}{2}}-\lambda^{2} A\right] \tilde{u}_{j+2 q+2},
\end{aligned}
$$

$j \in \mathbb{Z}$. Let

$$
\begin{aligned}
H & =\lambda A^{\frac{1}{2}}-\lambda^{2} A, \bar{A}=\frac{\lambda}{2} A^{\frac{1}{2}}(1+\kappa)+\frac{\lambda}{2} A^{\frac{1}{2}}+\frac{\lambda}{2} \kappa F^{\prime}(u), B=\lambda^{2} A-\lambda A^{\frac{1}{2}}, \\
D & =\left(1-A^{\frac{1}{2}} \lambda\right)(1+\kappa)+1-A^{\frac{1}{2}} \lambda, E=2 \lambda A^{\frac{1}{2}}-2 \lambda^{2} A-1, \\
F & =\frac{\lambda}{2} A^{\frac{1}{2}}(1+\kappa)+\frac{\lambda}{2} A^{\frac{1}{2}}-\frac{\lambda}{2} \kappa F^{\prime}(u), C=-(1+\kappa) .
\end{aligned}
$$


So we can rewrite (2.4) as

$$
\begin{aligned}
\tilde{u}_{2 q+2}= & H^{-1}\left[\frac{\lambda}{2} A^{\frac{1}{2}}(1+\kappa)+\frac{\lambda}{2} A^{\frac{1}{2}}\right] \tilde{u}_{2 q+1}+H^{-1} D \tilde{u}_{q+1}-H^{-1}[1+\kappa] \tilde{u}_{q} \\
& +H^{-1}\left[\frac{\lambda}{2} A^{\frac{1}{2}}(1+\kappa)+\frac{\lambda}{2} A^{\frac{1}{2}}\right] \tilde{u}_{1}+H^{-1} B \tilde{u}_{2} \\
& -\frac{\lambda}{2} \kappa H^{-1}\left[F\left(\tilde{u}_{2 q+1}\right)-F\left(\tilde{u}_{1}\right)\right]+H^{-1} E \tilde{u}_{q+2} \\
\equiv & G\left(\tilde{u}_{1}, \ldots, \tilde{u}_{2 q+1}\right) .
\end{aligned}
$$

Define the matching map associated with (2.4), $T: \mathbb{R}^{m(2 q+1)} \rightarrow \mathbb{R}^{m(2 q+1)}$, as

$$
T\left(\begin{array}{c}
\tilde{u}_{1} \\
\tilde{u}_{2} \\
\cdot \\
\cdot \\
\cdot \\
\tilde{u}_{2 q+1}
\end{array}\right)=\left(\begin{array}{c}
\tilde{u}_{2} \\
\tilde{u}_{3} \\
\cdot \\
\cdot \\
\cdot \\
G\left(\tilde{u}_{1}, \ldots, \tilde{u}_{2 q+1}\right)
\end{array}\right) .
$$

The notation $\mathbf{u}$ in $\mathbb{R}^{m(2 q+1)}$ is denoted by

$$
\mathbf{u}=\left(\begin{array}{c}
u \\
\cdot \\
\cdot \\
\cdot \\
u
\end{array}\right)
$$

for $u \in \mathbb{R}^{m}$ and let $\Delta=\left\{\mathbf{u} \mid u \in \mathbb{R}^{m}\right\}$. Using the nonlinear marching map $T$, it is obvious that proving the existence of the $u$ component discrete shock profiles is equivalent to finding the initial data

$$
\left(\begin{array}{c}
\widetilde{u}_{1} \\
\cdot \\
\cdot \\
\cdot \\
\widetilde{u}_{2 q+1}
\end{array}\right) \in \mathbb{R}^{m(2 q+1)}
$$

such that

$$
\begin{array}{r}
\lim _{j \rightarrow \infty} T^{j}\left(\begin{array}{c}
\widetilde{u}_{1} \\
\cdot \\
\cdot \\
\cdot \\
\widetilde{u}_{2 q+1}
\end{array}\right)=\mathbf{u}_{+}, \\
\lim _{j \rightarrow-\infty} T^{j}\left(\begin{array}{c}
\widetilde{u}_{1} \\
\cdot \\
\cdot \\
\cdot \\
\widetilde{u}_{2 q+1}
\end{array}\right)=\mathbf{u}_{-\cdot}
\end{array}
$$

We only need to check whether $T$ satisfies the five conditions at the beginning of this section.

(1) Obviously, $T \mathbf{u}=\mathbf{u}$. Let $\mu_{s} \equiv\left\{\mathbf{u} \mid \lambda_{k}(u)=s\right\}, \mathbf{u}_{\mathbf{0}} \in \mu_{s}, \nu$ denote a sufficiently small neighborhood of $\mathbf{u}_{\mathbf{0}}$. Under the subcharacteristic condition, it is clear that $T$ satisfies the first and second conditions. 
(2) Suppose that $\lambda_{k}\left(\mathbf{u}_{\mathbf{0}}\right)=s$ and $\lambda_{k}$ is genuinely nonlinear. Then the algebraic eigenspace of $d T\left(\mathbf{u}_{\mathbf{0}}\right)$ belonging to the eigenvalue 1 is $m+1$-dimensional, which is spanned by the vectors

$$
\left\{\mathbf{r}_{\mathbf{j}}\left(u_{0}\right)\right\}_{j=1}^{m} \cup\left\{e_{1}\left(u_{0}\right)\right\},
$$

where

$$
e_{1}^{t}\left(u_{0}\right)=\left(r_{k}\left(u_{0}\right), 2 r_{k}\left(u_{0}\right), \ldots,(2 q+1) r_{k}\left(u_{0}\right)\right),
$$

$\left(d T\left(\mathbf{u}_{\mathbf{0}}\right)\right) \mathbf{r}_{\mathbf{j}}=\mathbf{r}_{\mathbf{j}}$ and $d T\left(\mathbf{u}_{\mathbf{0}}\right) e_{1}=e_{1}+\mathbf{r}_{\mathbf{k}}$.

Proof. Since

$$
d T(\mathbf{u})=\left(\begin{array}{cccccccc}
0 & I & & & & & & \\
& 0 & I & & & & & \\
& & \cdot & . & \dot{1} & & \\
H^{-1} \bar{A} & H^{-1} B & \cdots & H^{-1} C & H^{-1} D & H^{-1} E & \cdots & H^{-1} F
\end{array}\right),
$$

the eigenvalues $\mathrm{Z}$ satisfy the equality

$$
\operatorname{det}\left[Z^{2 q} F-Z^{2 q+1} H+Z^{q+1} E+Z^{q} D+Z^{q-1} C+Z B+\bar{A}\right]=0 .
$$

When $Z=1$,

$$
F-H+E+D+C+B+\bar{A}=0
$$

so

$$
\operatorname{det}\left[(Z-1)(2 q F-(2 q+1) H+(q+1) E+q D+(q-1) C+B)+O(Z-1)^{2}\right]=0 ;
$$

i.e.,

$$
\operatorname{det}\left[(Z-1) \kappa\left(1-\lambda q F^{\prime}(u)\right)+O(Z-1)^{2}\right]=0
$$

Write

$$
\mho F^{\prime}(u) \mho^{-1}=\left(\begin{array}{ccccccc}
\lambda_{k} & & & & & & \\
& \lambda_{1} & & & & & \\
& & \ddots & & & & \\
& & & \lambda_{k-1} & & & \\
& & & \lambda_{k+1} & & \\
& & & & \ddots & \\
& & & & & \lambda_{m}
\end{array}\right),
$$

so

$$
(Z-1)^{m} \operatorname{det}\left[\mho \kappa\left(1-\lambda q F^{\prime}(u)\right) \mho^{-1}+O(Z-1)\right]=0,
$$

and when $\lambda_{k}\left(u_{0}\right)=s$, we have $1-\lambda q \lambda_{k}\left(u_{0}\right)=0$, and the first part follows. Since

$$
H^{-1} \bar{A}+H^{-1} B+H^{-1} C+H^{-1} D+H^{-1} E+H^{-1} F=I,
$$

it is easy to check that $d T(\mathbf{u}) \mathbf{r}_{j}=\mathbf{r}_{j}$ and

$$
d T\left(\mathbf{u}_{0}\right) e_{1}=\left(\begin{array}{c}
2 r_{k} \\
\vdots \\
(2 q+1) r_{k} \\
(2 q+2) r_{k}
\end{array}\right)=e_{1}+\mathbf{r}_{k}
$$


Remark 2.2. For $\lambda s=\frac{p}{q}$ and $s>0$, whatever the relations between $q$ and $p$, the eigenvalue equation will be

$$
\operatorname{det}\left[\bar{A}+Z^{q-p} C+Z^{p} B+Z^{q} D+Z^{q+p} E+Z^{2 q} F-Z^{2 q+p} H\right]=0 .
$$

Then we can check the condition (2) in exactly the same way. When $s<0$, we can also show this with a little modification.

(3) (Nonresonance condition) If $\lambda_{0}$ is sufficiently small, for $\lambda<\lambda_{0}$, there is a $\delta_{1}>0$ such that, for $\left|\mathbf{u}-\mathbf{u}_{0}\right|<\delta_{1}$, there is no eigenvalue of $d T(\mathbf{u})$ in the set $\{Z:|Z|=1, Z \neq 1\}$.

Proof. The eigenvalues must satisfy the equation

$$
\begin{aligned}
& Z^{2 q}\left(\frac{\lambda}{2} a^{\frac{1}{2}}(1+\kappa)+\frac{\lambda}{2} a^{\frac{1}{2}}-\frac{\lambda}{2} \kappa \lambda_{j}\right)-Z^{2 q+1}\left(\lambda a^{\frac{1}{2}}-\lambda^{2} a\right) \\
& +Z^{q+1}\left(2 \lambda a^{\frac{1}{2}}-2 \lambda^{2} a-1\right)+Z^{q}\left(\left(1-a^{\frac{1}{2}} \lambda\right)(2+\kappa)\right)-Z^{q-1}(1+\kappa) \\
& +Z\left(\lambda^{2} a-\lambda a^{\frac{1}{2}}\right)+\left(\frac{\lambda}{2} a^{\frac{1}{2}}(1+\kappa)+\frac{\lambda}{2} a^{\frac{1}{2}}+\frac{\lambda}{2} \kappa \lambda_{j}\right)=0,
\end{aligned}
$$

for $j=1, \ldots, m$. If there exist a $Z=e^{i \theta}$ for suitably small $\lambda$ satisfying the equation, we can divide the equation by $Z^{q-1}$ to get

$$
\begin{aligned}
& Z^{q+1}\left(\frac{\lambda}{2} a^{\frac{1}{2}}(1+\kappa)+\frac{\lambda}{2} a^{\frac{1}{2}}-\frac{\lambda}{2} \kappa \lambda_{j}\right)-Z^{q+2}\left(\lambda a^{\frac{1}{2}}-\lambda^{2} a\right) \\
& +Z^{2}\left(2 \lambda a^{\frac{1}{2}}-2 \lambda^{2} a-1\right)+Z\left(\left(1-a^{\frac{1}{2}} \lambda\right)(2+\kappa)\right)-(1+\kappa) \\
& +Z^{2-q}\left(\lambda^{2} a-\lambda a^{\frac{1}{2}}\right)+Z^{1-q}\left(\frac{\lambda}{2} a^{\frac{1}{2}}(1+\kappa)+\frac{\lambda}{2} a^{\frac{1}{2}}+\frac{\lambda}{2} \kappa \lambda_{j}\right)=0,
\end{aligned}
$$

for $j=1, \ldots, m$. Let $\lambda \rightarrow 0$. The equation will be

$$
Z^{2}-(2+\kappa) Z+1+\kappa=0
$$

It is impossible that $Z=e^{i \theta}$, so by perturbation analysis, $\exists \lambda_{0}$, for $\lambda<\lambda_{0}$, there is a $\delta_{1}>0$ such that $\left|u-u_{0}\right|<\delta_{1}$; eigenvalues do not belong to the set $\{Z:|Z|=1, Z \neq 1\}$.

Remark 2.3. If $\kappa$ is sufficiently large, divide the equation by $\kappa$, and let $\lambda \rightarrow 0$. We have the equation $Z=1$, which is a contradiction too.

Remark 2.4. For $\lambda s=\frac{p}{q}, s>0$, the eigenvalue equations are

$$
\begin{aligned}
& \frac{\lambda}{2} a^{\frac{1}{2}}(1+\kappa)+\frac{\lambda}{2} a^{\frac{1}{2}}+\frac{\lambda}{2} \kappa \lambda_{j}-(1+\kappa) Z^{q-p}+\left(\lambda^{2} a-\lambda a^{\frac{1}{2}}\right) Z^{p} \\
& \quad+\left(1-a^{\frac{1}{2}} \lambda\right)(2+\kappa) Z^{q}+\left(2 \lambda a^{\frac{1}{2}}-2 \lambda^{2} a-1\right) Z^{q+p} \\
& \quad+\left(\frac{\lambda}{2} a^{\frac{1}{2}}(1+\kappa)+\frac{\lambda}{2} a^{\frac{1}{2}}-\frac{\lambda}{2} \kappa \lambda_{j}\right) Z^{2 q}-\left(\lambda a^{\frac{1}{2}}-\lambda^{2} a\right) Z^{2 q+p}=0,
\end{aligned}
$$

for $j=1, \ldots, m$. Setting $\lambda \rightarrow 0$, we obtain the equation

$$
Z^{2 p}-(2+\kappa) Z^{p}+(1+\kappa)=0 .
$$


Thus $e^{i \frac{2 \pi n}{p}}$ are solutions if $|Z|=1, n \in Z$. Substituting these solutions into the original equation, we get the equation

$$
\begin{aligned}
\left(-2 \lambda^{2} a-\kappa a^{\frac{1}{2}} \lambda\right) e^{i \frac{2 q \pi n}{p}} & +\left(\frac{\lambda}{2} \kappa a^{\frac{1}{2}}+\lambda^{2} a-\frac{\lambda}{2} \kappa \lambda_{j}\right) e^{i \frac{4 q \pi n}{p}} \\
& +\left(\lambda^{2} a+\frac{\lambda}{2} a^{\frac{1}{2}} \kappa+\frac{\lambda}{2} \kappa \lambda_{j}\right)=0
\end{aligned}
$$

This is impossible if $n \neq k p$, so we get a contradiction.

(4) There are $\delta_{2}>0$ and $r_{2}>0$ such that, for $\left|\mathbf{u}-\mathbf{u}_{0}\right|<\delta_{2}$, there are only $m+1$ eigenvalues of $d T(\mathbf{u})$ lying in $|Z-1|<r_{2}$. These eigenvalues are given by $1,1, \ldots, 1, \beta(u)$, and $\beta(u)$ is real and satisfies

(a) $\beta(u)=1$ whenever $\lambda_{k}(u)=s$,

(b) $\left.\nabla_{u} \beta \cdot r_{k}(u)\right|_{u=u_{0}}>0$ under the subcharacteristic and CFL conditions.

Proof. The first statement is guaranteed by (2) and (3), and (a) comes from (2) immediately. Assume that $q>1$ (the case $q=1$ is similar). Then

$$
\operatorname{det}\left[\mho \kappa\left(1-\lambda q F^{\prime}(u)\right) \mho^{-1}+R(\beta(u)-1)+O(\beta(u)-1)^{2}\right]=0
$$

where

$$
\begin{aligned}
R= & 2 q(2 q-1)\left(\frac{\lambda}{2} A^{\frac{1}{2}}(1+\kappa)+\frac{\lambda}{2} A^{\frac{1}{2}}-\frac{\lambda}{2} \kappa \Lambda\right)-(2 q+1) 2 q\left(\lambda A^{\frac{1}{2}}-\lambda^{2} A\right) \\
& +(q+1) q\left(2 \lambda A^{\frac{1}{2}}-2 \lambda^{2} A-1\right)+q(q-1)\left(1-A^{\frac{1}{2}}\right)(2+\kappa) \\
& -(q-1)(q-2)(1+\kappa) .
\end{aligned}
$$

Now expanding along the first row, one gets that

$$
\begin{aligned}
\kappa(1 & \left.-\lambda q \lambda_{k}(u)\right)+\left[q^{2} \lambda a^{\frac{1}{2}} \kappa-2 q^{2} \lambda \kappa \lambda_{k}(u)+2 q^{2} \lambda^{2} a+2 q \kappa-2-2 \kappa\right. \\
& \left.+q \lambda \kappa \lambda_{k}(u)\right](\beta(u)-1)+O(\beta(u)-1)^{2}=0
\end{aligned}
$$

Set

$$
\tilde{\tau_{1}}=q^{2} \lambda a^{\frac{1}{2}} \kappa+2 q^{2} \lambda^{2} a-2-k .
$$

Then $\tilde{\tau}_{1}>0$ under the subcharacteristic and CFL conditions. Thus the coefficient of $(\beta(u)-1)$ is positive when $u=u_{0}$ in equation (2.6). By the implicit function theorem, $\exists \delta_{2}$, when $\left|\mathbf{u}-\mathbf{u}_{0}\right|<\delta_{2}$, there is a unique real smooth function $\beta(u)$ with $\beta\left(u_{0}\right)=1$ provided that $\lambda_{k}\left(u_{0}\right)=s$. From equation (2.6), we have

$$
\left.\nabla_{u} \beta(u) \cdot r_{k}\right|_{u=u_{0}}=\left.\frac{\lambda q \kappa \nabla_{u} \lambda_{k} \cdot r_{k}}{\tilde{\tau_{1}}}\right|_{u=u_{0}} .
$$

Remark 2.5. For $\lambda s=\frac{p}{q}, s>0$, equation (2.6) becomes

$$
\begin{aligned}
\kappa(p & \left.-\lambda q \lambda_{k}(u)\right)+\left[\left(2 q p-p^{2}-p\right) \kappa-2 p^{2}-q(2 q-1) \lambda \kappa \lambda_{k}(u)+2 q^{2} \lambda^{2} a\right. \\
& \left.+q^{2} \kappa \lambda a^{\frac{1}{2}}\right](\beta(u)-1)+O(\beta(u)-1)^{2}=0
\end{aligned}
$$

When $u=u_{0}$, the coefficient of $\beta(u)$ is also positive under the subcharacteristic and CFL conditions. Thus we can prove the existence of the real function $\beta(u)$. 
Remark 2.6. $\tilde{\tau_{1}}>0$ and equation (2.6) are crucial for showing the strict monotonicity of the principal characteristic speed at the discrete shock profile and the asymptotic behavior of the discrete shock profiles. This monotonicity is crucial to our stability analysis (see [16]).

Remark 2.7. We need to assume that $\lambda$ is suitably small, and it also implies that $q$ is suitably large.

So, we have established the existence of entropy satisfying discrete shock profiles of the $u$-component. Furthermore, by Proposition 2.1 of Liu-Xin in [16], we have the following proposition.

Proposition 2.2. There exists a family of discrete shock profiles $\phi_{j}^{n}$ of (2.4) connecting $u_{-}$and $u_{+}$, provided that $u_{ \pm}$satisfy the entropy condition, $\epsilon=\left|u_{-}-u_{+}\right|$ is suitably small and the subcharacteristic condition is satisfied. Furthermore, the discrete shock profiles $\phi_{j}^{n}$ have the following properties for $j=0, \pm 1, \ldots$ :

$$
\begin{gathered}
\lambda_{k}\left(\phi_{j}\right)>\lambda_{k}\left(\phi_{j+1}\right), \\
\left|\lambda_{k}\left(\phi_{j}\right)-s\right| \leq c_{1} \epsilon, \\
c_{2}\left|\phi_{j}-\phi_{j+1}\right| \leq \lambda_{k}\left(\phi_{j}\right)-\lambda_{k}\left(\phi_{j+1}\right) \leq c_{3}\left|\phi_{j}-\phi_{j+1}\right|, \\
\nabla F\left(\phi_{j}\right)\left(\phi_{j+1}-\phi_{j}\right)=s\left(\phi_{j+1}-\phi_{j}\right)+O(\epsilon)\left|\phi_{j+1}-\phi_{j}\right|, \\
\left|\phi_{j+1}-2 \phi_{j}+\phi_{j-1}\right| \leq c_{4} \epsilon\left|\phi_{j+1}-\phi_{j}\right|, \\
c_{5} \epsilon^{2} e^{-c_{6} \epsilon|j|} \leq \lambda_{k}\left(\phi_{j}\right)-\lambda_{k}\left(\phi_{j+1}\right) \leq c_{7} \epsilon^{2} e^{-c_{8} \epsilon|j|}, \\
c_{9} \epsilon e^{-c_{10} \epsilon|j|} \leq\left|\phi_{j}-u^{ \pm}\right| \leq c_{11} \epsilon e^{-c_{12} \epsilon|j|}, \\
c_{12}\left|\phi_{j}-\phi_{j+q}\right| \leq \lambda_{k}\left(\phi_{j}\right)-\lambda_{k}\left(\phi_{j+q}\right) \leq c_{13}\left|\phi_{j}-\phi_{j+q}\right|, \\
\nabla F\left(\phi_{j}\right)\left(\phi_{j+q}-\phi_{j}\right)=s\left(\phi_{j+q}-\phi_{j}\right)+O(\epsilon)\left|\phi_{j+q}-\phi_{j}\right|, \\
\left|\phi_{j+q}-2 \phi_{j}+\phi_{j-q}\right| \leq c_{14} \epsilon\left|\phi_{j+q}-\phi_{j}\right|
\end{gathered}
$$

where $c_{1}, \ldots, c_{14}$ are positive constants independent of $\epsilon$ and $j$.

Combining the above proposition and following Liu, Wang and Yang [13, we can also find the discrete shock profiles of the $v$ component. To this end, summing the first equation of (1.3) over $x$ from $y-2 N+1$ to $y-1$ with step size (2), we have

$$
\begin{aligned}
v_{y-2 N}= & v_{y}+\frac{2}{\lambda} \sum_{x=y-2 N+1}^{y-1}\left(u_{x-\eta}-u_{x}\right) \\
& +A^{\frac{1}{2}} \sum_{x=y-2 N+1}^{y-1}\left[\left(u_{x}-u_{x-1}\right)-\left(u_{x+1}-u_{x}\right)\right] .
\end{aligned}
$$

Since $\left|u_{x-x_{0}}-u_{x}\right|<c\left(\lambda_{k}\left(u_{x-x_{0}}\right)-\lambda_{k}\left(u_{x}\right)\right)$ and $\sum_{x=-\infty}^{y_{0}}\left(\lambda_{k}\left(u_{x-x_{0}}\right)-\lambda_{k}\left(u_{x}\right)\right)<$ $O(1) \epsilon$, then $\sum_{x=-\infty}^{y_{0}}\left(u_{x-x_{0}}-u_{x}\right)$ converges. Hence, $v_{y-2 N}$ converges as $N \rightarrow \infty$ 
for any $y$, which implies that $\lim _{x \rightarrow \pm \infty} v_{x}=F\left(u_{ \pm}\right)$. Thus $v_{x}$ can be expressed as

$$
\begin{aligned}
v_{x}= & v_{-}+\frac{2}{\lambda} \sum_{m=-\infty}^{0}\left(u_{x+2 m-1}-u_{x+2 m-1-\eta}\right) \\
& +A^{\frac{1}{2}} \sum_{m=-\infty}^{0}\left(u_{x+2 m}-2 u_{x+2 m-1}+u_{x+2 m-2}\right) .
\end{aligned}
$$

Similarly, as for Corollary 2.1 of Liu and Xin [16], we can also have the following theorem.

Theorem 2.3. There exists a family of discrete shock profiles $\left(\phi_{j}^{n}, \psi_{j}^{n}\right)$ of (1.4), connecting $\left(u_{-}, f\left(u_{-}\right)\right)$and $\left(u_{+}, f\left(u_{+}\right)\right)$, provided that $u_{ \pm}$satisfy the entropy condition, $\epsilon=\left|u_{-}-u_{+}\right|$is suitably small and the subcharacteristic condition is satisfied. Furthermore, the discrete shock profiles $\phi_{j}^{n}$ have the following properties for $j=0, \pm 1, \ldots$ and $n=0,1, \ldots$ :

$$
\begin{gathered}
\lambda_{k}\left(\phi_{j}^{n}\right)>\lambda_{k}\left(\phi_{j+1}^{n}\right), \\
\left|\lambda_{k}\left(\phi_{j}^{n}\right)-s\right| \leq C_{1} \epsilon, \\
C_{2}\left|\phi_{j}^{n}-\phi_{j+1}^{n}\right| \leq \lambda_{k}\left(\phi_{j}^{n}\right)-\lambda_{k}\left(\phi_{j+1}^{n}\right) \leq C_{3}\left|\phi_{j}^{n}-\phi_{j+1}^{n}\right|, \\
\nabla F\left(\phi_{j}^{n}\right)\left(\phi_{j+1}^{n}-\phi_{j}^{n}\right)=s\left(\phi_{j+1}^{n}-\phi_{j}^{n}\right)+O(\epsilon)\left|\phi_{j+1}^{n}-\phi_{j}^{n}\right|, \\
\left|\phi_{j+1}^{n}-2 \phi_{j}^{n}+\phi_{j-1}^{n}\right| \leq C_{4} \epsilon\left|\phi_{j+1}^{n}-\phi_{j}^{n}\right|, \\
C_{5} \epsilon^{2} e^{-c_{6} \epsilon|j-\lambda s n|} \leq \lambda_{k}\left(\phi_{j}^{n}\right)-\lambda_{k}\left(\phi_{j+1}^{n}\right) \leq C_{7} \epsilon^{2} e^{-c 8 \epsilon|j-\lambda s n|,} \\
C_{9} \epsilon e^{-c_{10} \epsilon|j-\lambda s n|} \leq\left|\phi_{j}^{n}-u \pm\right| \leq C_{11} \epsilon e^{-c_{12} \epsilon|j-\lambda s n|,} \\
\left|\phi_{j}^{n \pm 1}-\phi_{j}^{n}\right| \leq C_{13}\left(\lambda_{k}\left(\phi_{j}^{n}\right)-\lambda_{k}\left(\phi_{j+1}^{n}\right)\right), n>1,
\end{gathered}
$$

where $C_{1}, \ldots, C_{13}$ are positive constants independent of $\epsilon, j$ and $n$.

Remark 2.8. It is not difficult to show that there exists a family of stationary discrete shock profiles and these profiles satisfy the above properties too.

\section{3. $L^{2}$ STABILITY OF STATIONARY DisCRETE SHOCK PROFILES}

In this section, we will prove Theorem 1.1. Let $\left(u_{j}^{n}, v_{j}^{n}\right)$ be a solution of the relaxing scheme with initial data satisfying (1.5)-(1.7), which is assumed to exist up to $n \leq n_{1}<+\infty$. Denote the stationary discrete shock profile $(\phi, \psi)$ as

$$
\begin{gathered}
\frac{\lambda}{2}\left(\psi_{j+1}-\psi_{j}\right)-\frac{\lambda}{2} A^{\frac{1}{2}}\left(\phi_{j+1}-2 \phi_{j}+\phi_{j-1}\right)=0, \\
\frac{\lambda}{2} A\left(\phi_{j+1}-\phi_{j-1}\right)-\frac{\lambda}{2} A^{\frac{1}{2}}\left(\psi_{j+1}-2 \psi_{j}+\psi_{j-1}\right)=\kappa\left(F\left(\phi_{j}\right)-\psi_{j}\right), \\
\lim _{j \rightarrow \pm \infty}\left(\phi_{j}, \psi_{j}\right)=\left(u_{ \pm}, f\left(u_{ \pm}\right)\right) .
\end{gathered}
$$

Let

$$
\bar{u}_{j}=u_{j}-\phi_{j}, \bar{v}_{j}=v_{j}-\psi_{j}
$$


Then

$$
\begin{array}{r}
\bar{u}_{j}^{n+1}-\bar{u}_{j}^{n}+\frac{\lambda}{2}\left(\bar{v}_{j+1}^{n}-\bar{v}_{j-1}^{n}\right)-\frac{\lambda}{2} A^{\frac{1}{2}}\left(\bar{u}_{j+1}^{n}-2 \bar{u}_{j}^{n}+\bar{u}_{j-1}^{n}\right)=0, \\
\bar{v}_{j}^{n+1}-\bar{v}_{j}^{n}+\frac{\lambda}{2} A\left(\bar{u}_{j+1}^{n}-\bar{u}_{j-1}^{n}\right)-\frac{\lambda}{2} A^{\frac{1}{2}}\left(\bar{v}_{j+1}^{n}-2 \bar{v}_{j}^{n}+\bar{v}_{j-1}^{n}\right) \\
=\kappa\left(\nabla F\left(\phi_{j}\right) \bar{u}_{j}^{n+1}-\bar{v}_{j}^{n+1}\right)+\kappa Q\left(u_{j}^{n+1}, \phi_{j}\right) .
\end{array}
$$

Let $Q\left(u_{j}^{n+1}, \phi_{j}\right)=F\left(u_{j}^{n+1}\right)-F\left(\phi_{j}\right)-\nabla F\left(\phi_{j}\right) \bar{u}_{j}^{n+1}$. Then $\left|Q\left(u_{j}^{n+1}, \phi_{j}\right)\right| \leq$ $O(1)\left|\bar{u}_{j}^{n+1}\right|^{2}$ when $u$ is in any bounded set. Formally let $\hat{u}_{j}^{n}=\sum_{i=-\infty}^{j} \bar{u}_{i}^{n}$, so $\bar{u}_{j}^{n}=\hat{u}_{j}^{n}-\hat{u}_{j-1}^{n}$ and

$$
P_{j}^{n} \equiv-\frac{\lambda}{2}\left(\bar{v}_{j+1}^{n}+\bar{v}_{j}^{n}\right)=\hat{u}_{j}^{n+1}-\hat{u}_{j}^{n}-\frac{\lambda}{2} A^{\frac{1}{2}}\left(\hat{u}_{j+1}^{n}-2 \hat{u}_{j}^{n}+\hat{u}_{j-1}^{n}\right) .
$$

Immediately, we have

$$
\begin{aligned}
P_{j}^{n+1} & -P_{j}^{n}+\frac{\kappa}{1+\kappa} P_{j}^{n}-\frac{\lambda^{2} A}{4(1+\kappa)}\left(\hat{u}_{j+2}^{n}-2 \hat{u}_{j}^{n}+\hat{u}_{j-2}^{n}\right) \\
& -\frac{\lambda A^{\frac{1}{2}}}{2(1+\kappa)}\left(P_{j+1}^{n}-2 P_{j}^{n}+P_{j-1}^{n}\right) \\
& +\frac{\lambda \kappa}{2(1+\kappa)} \nabla F\left(\phi_{j}\right)\left(\hat{u}_{j}^{n+1}-\hat{u}_{j-1}^{n+1}\right)+\frac{\lambda \kappa}{2(1+\kappa)} \nabla F\left(\phi_{j+1}\right)\left(\hat{u}_{j+1}^{n+1}-\hat{u}_{j}^{n+1}\right) \\
& =-\frac{\lambda}{2(1+\kappa)}\left[\kappa Q\left(u_{j}^{n+1}, \phi_{j}\right)+\kappa Q\left(u_{j+1}^{n+1}, \phi_{j+1}\right)\right] .
\end{aligned}
$$

Use the notation

$$
\left.L_{j}=L_{(} \phi_{j}\right), \Lambda_{j}=\Lambda\left(\phi_{j}\right), R_{j}=R\left(\phi_{j}\right), \theta_{j}^{n}=Q\left(u_{j}^{n}, \phi_{j}\right)
$$

We rewrite the system (3.2) in terms of the characteristic variables

$$
P_{j}^{n}=R_{j} \tilde{P}_{j}^{n}, \hat{u}_{j}^{n}=R_{j} \tilde{u}_{j}^{n}
$$

and letting $\mu=\lambda a^{\frac{1}{2}}$, we have the equation

$$
\begin{aligned}
\tilde{P}_{j}^{n+1} & -\tilde{P}_{j}^{n}+\frac{\kappa}{1+\kappa} \tilde{P}_{j}^{n}-\frac{\mu^{2}}{4(1+\kappa)}\left(\tilde{u}_{j+2}^{n}-2 \tilde{u}_{j}^{n}+\tilde{u}_{j-2}^{n}\right) \\
& -\frac{\mu}{2(1+\kappa)}\left(\tilde{P}_{j+1}^{n}-2 \tilde{P}_{j}^{n}+\tilde{P}_{j-1}^{n}\right) \\
& +\frac{\lambda \kappa}{2(1+\kappa)} \Lambda_{j}\left(\tilde{u}_{j}^{n+1}-\tilde{u}_{j-1}^{n+1}\right)+\frac{\lambda \kappa}{2(1+\kappa)} \Lambda_{j+1}\left(\tilde{u}_{j+1}^{n+1}-\tilde{u}_{j}^{n+1}\right) \\
& =\frac{\lambda \kappa}{2(1+\kappa)} \Lambda_{j}\left(L_{j+1}-L_{j-1}\right) R_{j} \tilde{u}_{j}^{n+1}+e_{j}^{n},
\end{aligned}
$$


where

$$
\begin{aligned}
e_{j}^{n}= & \frac{\lambda \kappa}{2(1+\kappa)} \Delta_{+} \Lambda_{j} \Delta_{0} L_{j} R_{j} \tilde{u}_{j}^{n+1}+\frac{\mu^{2}}{4(1+\kappa)} L_{j}\left(R_{j+2}-2 R_{j}+R_{j-2}\right) \tilde{u}_{j}^{n} \\
& -\frac{\lambda \kappa}{2(1+\kappa)} L_{j} \Delta_{+} R_{j} \Lambda_{j+1} \Delta_{+} \tilde{u}_{j}^{n+1}+\frac{\mu}{2(1+\kappa)} L_{j} \Delta R_{j} \tilde{P}_{j}^{n} \\
& -\frac{\lambda \kappa}{2(1+\kappa)} \Lambda_{j} \Delta_{-} L_{j} R_{j-1} \Delta_{-} \tilde{u}_{j}^{n+1}+\frac{\mu^{2}}{4(1+\kappa)} L_{j}\left(R_{j}-R_{j-2}\right)\left(\tilde{u}_{j}^{n}-\tilde{u}_{j-2}^{n}\right) \\
& +\frac{\mu^{2}}{4(1+\kappa)} L_{j}\left(R_{j+2}-R_{j}\right)\left(\tilde{u}_{j+2}^{n}-\tilde{u}_{j}^{n}\right)-\frac{\lambda \kappa}{2(1+\kappa)} \Lambda_{j} \Delta_{-} L_{j} \Delta_{-} R_{j} \tilde{u}_{j}^{n+1} \\
& +\frac{\mu}{2(1+\kappa)} L_{j} \Delta_{+} R_{j} \Delta_{+} \tilde{P}_{j}^{n}+\frac{\mu}{2(1+\kappa)} L_{j} \Delta_{-} R_{j} \Delta_{-} \tilde{P}_{j}^{n} \\
& +\frac{\lambda \kappa}{2(1+\kappa)} L_{j} \Delta_{+} R_{j} \Lambda_{j+1} \Delta_{+} L_{j} R_{j} \tilde{u}_{j}^{n+1}-\frac{\kappa \lambda}{2(1+\kappa)} L_{j}\left(\theta_{j}^{n+1}+\theta_{j+1}^{n+1}\right) .
\end{aligned}
$$

Here, we have used the notation

$$
\begin{aligned}
\Delta_{+} f_{j} & =f_{j+1}-f_{j}, \\
\Delta_{-} f_{j} & =f_{j}-f_{j-1}, \\
\Delta_{0} f_{j} & =f_{j+1}-f_{j-1}, \\
\Delta f_{j} & =f_{j+1}-2 f_{j}+f_{j-1}, \\
\Delta^{+} f^{n} & =f^{n+1}-f^{n} .
\end{aligned}
$$

Thus, to prove Theorem 1.1, we need to derive the basic prior $L^{2}$ estimate of the solution of (3.4). Denote the weight as $W_{j}$ which will be defined later. It is used so the terms of the principal direction control the transverse terms. Now we need some basic energy estimates. Assume $2 \tilde{P}_{j}^{n} W_{j} \times(\underline{3.4})$, and denote $\left|u_{j}^{n}\right|_{w}=\left(u_{j}^{n} . W_{j} u_{j}^{n}\right)^{\frac{1}{2}}$, because

$$
\begin{aligned}
\sum_{j} 2 \tilde{P}_{j}^{n} W_{j}\left(\tilde{P}_{j}^{n+1}-\tilde{P}_{j}^{n}\right) & \\
= & \sum_{j}\left|\tilde{P}_{j}^{n+1}\right|_{w}^{2}-\sum_{j}\left|\tilde{P}_{j}^{n}\right|_{w}^{2}-\sum_{j}\left|\tilde{P}_{j}^{n+1}-\tilde{P}_{j}^{n}\right|_{w}^{2}, \\
\sum_{j}- & \frac{\mu^{2}}{2(1+\kappa)} \tilde{P}_{j}^{n} W_{j}\left(\tilde{u}_{j+2}^{n}-2 \tilde{u}_{j}^{n}+\tilde{u}_{j-2}^{n}\right) \\
= & \frac{\mu^{2}}{2(1+\kappa)} \sum_{j} \tilde{P}_{j+1}^{n}\left(W_{j+1}-W_{j-1}\right)\left(\tilde{u}_{j+1}^{n}-\tilde{u}_{j-1}^{n}\right) \\
& +\frac{\mu^{2}}{2(1+\kappa)} \sum_{j}\left(\tilde{P}_{j+1}^{n}-\tilde{P}_{j-1}^{n}\right) W_{j-1}\left(\tilde{u}_{j+1}^{n}-\tilde{u}_{j-1}^{n}\right)
\end{aligned}
$$




$$
\begin{aligned}
\sum_{j}- & \frac{\mu}{1+\kappa} \tilde{P}_{j}^{n} W_{j}\left(\tilde{P}_{j+1}-2 \tilde{P}_{j}+\tilde{P}_{j-1}\right) \\
= & \frac{\mu}{2(1+\kappa)} \sum_{j}\left(\tilde{P}_{j+1}^{n}-\tilde{P}_{j}^{n}\right)\left(W_{j}+W_{j+1}\right)\left(\tilde{P}_{j+1}^{n}-\tilde{P}_{j}^{n}\right) \\
& -\frac{\mu}{2(1+\kappa)} \sum_{j} \tilde{P}_{j}^{n}\left(W_{j+1}-2 W_{j}+W_{j-1}\right) \tilde{P}_{j}^{n} .
\end{aligned}
$$

Let

$$
M_{j}^{n}=\tilde{u}_{j+1}^{n}-\tilde{u}_{j}^{n}, N_{j}^{n}=\tilde{u}_{j+1}^{n}-\tilde{u}_{j-1}^{n} .
$$

After summation by parts,

$$
\begin{aligned}
\sum_{j}\left|\tilde{P}_{j}^{n+1}\right|_{w}^{2}-\sum_{j}\left|\tilde{P}_{j}^{n}\right|_{w}^{2} \\
+\frac{2 \kappa}{1+\kappa} \sum_{j}\left|\tilde{P}_{j}^{n}\right|_{w}^{2} \\
+\frac{\mu^{2}}{2(1+\kappa)} \sum_{j} \tilde{P}_{j+1}^{n} \Delta_{0} W_{j} N_{j}^{n} \\
+\frac{\mu}{2(1+\kappa)} \sum_{j}\left(\tilde{P}_{j+1}^{n}-\tilde{P}_{j}^{n}\right)\left(W_{j}+W_{j+1}\right)\left(\tilde{P}_{j+1}^{n}-\tilde{P}_{j}^{n}\right) \\
=\sum_{j}\left|\tilde{P}_{j}^{n+1}-\tilde{P}_{j}^{n}\right|_{w}^{2} \\
\quad-\frac{\mu^{2}}{2(1+\kappa)} \sum_{j}\left(\tilde{P}_{j+1}^{n}-\tilde{P}_{j-1}^{n}\right) W_{j-1} N_{j}^{n} \\
+\frac{\mu}{2(1+\kappa)} \sum_{j} \tilde{P}_{j}^{n} \Delta W_{j} \tilde{P}_{j}^{n} \\
\quad-\sum_{j} \frac{\lambda \kappa}{1+\kappa}\left(\tilde{P}_{j+1}^{n} W_{j+1}+\tilde{P}_{j}^{n} W_{j}\right) \Lambda_{j+1}\left(\tilde{u}_{j+1}^{n+1}-\tilde{u}_{j}^{n+1}\right) \\
+\sum_{j} \frac{\lambda \kappa}{1+\kappa} \tilde{P}_{j}^{n} W_{j} \Lambda_{j}\left(L_{j+1}-L_{j-1}\right) R_{j} \tilde{u}_{j}^{n+1} \\
+\sum_{j} 2 \tilde{P}_{j}^{n} W_{j} e_{j}^{n} .
\end{aligned}
$$

Because of (3.1), we have the identities

$$
\begin{aligned}
\tilde{u}_{j}^{n+1}= & (1-\mu) \tilde{u}_{j}^{n}+\tilde{P}_{j}^{n}+\frac{\mu}{2} L_{j} R_{j+1} \tilde{u}_{j+1}^{n}+\frac{\mu}{2} L_{j} R_{j-1} \tilde{u}_{j-1}^{n}, \\
\tilde{u}_{j+1}^{n+1}-\tilde{u}_{j}^{n+1}= & (1-\mu) M_{j}^{n}+\tilde{P}_{j+1}^{n}-\tilde{P}_{j}^{n} \\
& +\frac{\mu}{2}\left(L_{j+1} R_{j+2} \tilde{u}_{j+2}^{n}-L_{j} R_{j+1} \tilde{u}_{j+1}^{n}\right) \\
& +\frac{\mu}{2}\left(L_{j+1} R_{j} \tilde{u}_{j}^{n}-L_{j} R_{j-1} \tilde{u}_{j-1}^{n}\right) .
\end{aligned}
$$


Next, assume $2 \tilde{u}_{j}^{n+1} W_{j} \times(3.4$, because of the identities

$$
\begin{aligned}
\sum_{j} \frac{2 \kappa}{1+\kappa} \tilde{u}_{j}^{n+1} W_{j} \tilde{P}_{j}^{n} \\
=\sum_{j} \frac{2 \kappa}{1+\kappa} \tilde{u}_{j}^{n+1} W_{j}\left(\tilde{u}_{j}^{n+1}-\tilde{u}_{j}^{n}-\frac{\mu}{2} L_{j}\left(R_{j+1} \tilde{u}_{j+1}^{n}-2 R_{j} \tilde{u}_{j}^{n}+R_{j-1} \tilde{u}_{j-1}^{n}\right)\right) \\
=\sum_{j} \frac{\kappa}{1+\kappa} \tilde{u}_{j}^{n+1} W_{j} \tilde{u}_{j}^{n+1}-\sum_{j} \frac{\kappa}{1+\kappa} \tilde{u}_{j}^{n} W_{j} \tilde{u}_{j}^{n} \\
\quad+\sum_{j} \frac{\kappa}{1+\kappa} \Delta^{+} \tilde{u}_{j}^{n} W_{j} \triangle^{+} \tilde{u}_{j}^{n} \\
\quad-\frac{\kappa \mu}{1+\kappa} \sum_{j} \Delta^{+} \tilde{u}_{j}^{n} W_{j} L_{j}\left(R_{j+1} \tilde{u}_{j+1}^{n}-2 R_{j} \tilde{u}_{j}^{n}+R_{j-1} \tilde{u}_{j-1}^{n}\right) \\
\quad+\frac{\kappa \mu}{2(1+\kappa)} \sum_{j} \Delta_{+} \tilde{u}_{j}^{n}\left(W_{j}+W_{j+1}\right) \Delta_{+} \tilde{u}_{j}^{n} \\
\quad-\frac{\kappa \mu}{2(1+\kappa)} \sum_{j} \tilde{u}_{j}^{n} \Delta W_{j} \tilde{u}_{j}^{n} \\
\quad-\frac{\kappa \mu}{1+\kappa} \sum_{j} \tilde{u}_{j}^{n} W_{j} L_{j} \Delta_{+} R_{j} \Delta_{+} \tilde{u}_{j}^{n} \\
\quad-\frac{\kappa \mu}{1+\kappa} \sum_{j} \tilde{u}_{j}^{n} W_{j} L_{j} \Delta_{-} R_{j} \Delta_{-} \tilde{u}_{j}^{n} \\
\quad-\frac{\kappa \mu}{1+\kappa} \sum_{j} \tilde{u}_{j}^{n} W_{j} L_{j} \Delta R_{j} \tilde{u}_{j}^{n}
\end{aligned}
$$

and

$$
\begin{aligned}
\sum_{j}- & \frac{\mu^{2}}{2(1+\kappa)} \tilde{u}_{j}^{n+1} W_{j}\left(N_{j+1}^{n}-N_{j-1}^{n}\right) \\
= & -\frac{\mu^{2}}{2(1+\kappa)} \sum_{j} \Delta^{+} \tilde{u}_{j}^{n} W_{j} \Delta_{0} N_{j}^{n}+\frac{\mu^{2}}{2(1+\kappa)} \sum_{j} N_{j}^{n} W_{j} N_{j}^{n} \\
& +\frac{\mu^{2}}{2(1+\kappa)} \sum_{j} \tilde{u}_{j+1}^{n} \Delta_{+} W_{j} N_{j}^{n}+\frac{\mu^{2}}{2(1+\kappa)} \sum_{j} \tilde{u}_{j-1}^{n} \Delta_{-} W_{j} N_{j}^{n}, \\
\sum_{j}- & \frac{\mu}{1+\kappa} \tilde{u}_{j}^{n+1} W_{j} \Delta \tilde{P}_{j}^{n} \\
= & \sum_{j}-\frac{\mu}{1+\kappa} \Delta^{+} \tilde{u}_{j}^{n} W_{j} \Delta \tilde{P}_{j}^{n} \\
& +\sum_{j} \frac{\mu}{1+\kappa} \Delta_{+} \tilde{u}_{j}^{n} W_{j} \Delta_{+} \tilde{P}_{j}^{n}+\sum_{j} \frac{\mu}{1+\kappa} \tilde{u}_{j+1}^{n} \Delta_{+} W_{j} \Delta_{+} \tilde{P}_{j}^{n} .
\end{aligned}
$$


Combining these terms and performing summation by parts, we get

$$
\begin{aligned}
& 2 \sum_{j} \tilde{u}_{j}^{n+1} W_{j} \tilde{P}_{j}^{n+1}-2 \sum_{j} \tilde{u}_{j}^{n} W_{j} \tilde{P}_{j}^{n} \\
& -2 \sum_{j} \Delta^{+} \tilde{u}_{j}^{n} W_{j} \tilde{P}_{j}^{n} \\
& +\sum_{j} \frac{\kappa}{1+\kappa} \tilde{u}_{j}^{n+1} W_{j} \tilde{u}_{j}^{n+1}-\sum_{j} \frac{\kappa}{1+\kappa} \tilde{u}_{j}^{n} W_{j} \tilde{u}_{j}^{n} \\
& +\sum_{j} \frac{\kappa}{1+\kappa} \Delta^{+} \tilde{u}_{j}^{n} W_{j} \Delta^{+} \tilde{u}_{j}^{n} \\
& -\frac{\kappa \mu}{1+\kappa} \sum_{j} \Delta^{+} \tilde{u}_{j}^{n} W_{j} L_{j}\left(R_{j+1} \tilde{u}_{j+1}^{n}-2 R_{j} \tilde{u}_{j}^{n}+R_{j-1} \tilde{u}_{j-1}^{n}\right) \\
& -\frac{\kappa \mu}{1+\kappa} \sum_{j} \tilde{u}_{j}^{n} W_{j} L_{j} \Delta_{+} R_{j} \Delta_{+} \tilde{u}_{j}^{n} \\
& -\frac{\kappa \mu}{1+\kappa} \sum_{j} \tilde{u}_{j}^{n} W_{j} L_{j} \Delta_{-} R_{j} \Delta_{-} \tilde{u}_{j}^{n} \\
& +\frac{\kappa \mu}{2(1+\kappa)} \sum_{j} \Delta_{+} \tilde{u}_{j}^{n}\left(W_{j}+W_{j+1}\right) \Delta_{+} \tilde{u}_{j}^{n} \\
& -\frac{\kappa \mu}{2(1+\kappa)} \sum_{j} \tilde{u}_{j}^{n} \Delta W_{j} \tilde{u}_{j}^{n} \\
& -\frac{\mu^{2}}{2(1+\kappa)} \sum_{j} \Delta^{+} \tilde{u}_{j}^{n} W_{j} \Delta_{0} N_{j}^{n}+\frac{\mu^{2}}{2(1+\kappa)} \sum_{j} N_{j}^{n} W_{j} N_{j}^{n} \\
& +\frac{\mu^{2}}{2(1+\kappa)} \sum_{j} \tilde{u}_{j+1}^{n} \Delta_{+} W_{j} N_{j}^{n}+\frac{\mu^{2}}{2(1+\kappa)} \sum_{j} \tilde{u}_{j-1}^{n} \Delta_{-} W_{j} N_{j}^{n} \\
& +\sum_{j} \frac{\mu}{1+\kappa} \Delta_{+} \tilde{u}_{j}^{n} W_{j} \Delta_{+} \tilde{P}_{j}^{n}+\sum_{j} \frac{\mu}{1+\kappa} \tilde{u}_{j+1}^{n} \Delta_{+} W_{j} \Delta_{+} \tilde{P}_{j}^{n} \\
& +\sum_{j} \frac{\lambda \kappa}{1+\kappa}\left(\tilde{u}_{j+1}^{n+1} W_{j+1}+\tilde{u}_{j}^{n+1} W_{j}\right) \Lambda_{j+1} \Delta_{+} \tilde{u}_{j}^{n+1} \\
& -\frac{\kappa \mu}{1+\kappa} \sum_{j} \tilde{u}_{j}^{n} W_{j} L_{j} \Delta R_{j} \tilde{u}_{j}^{n} \\
& -\sum_{j} \frac{\mu}{1+\kappa} \Delta^{+} \tilde{u}_{j}^{n} W_{j} \Delta \tilde{P}_{j}^{n} \\
& \tilde{u}_{j}^{n+1} W_{j} \Lambda_{j} \Delta_{0} L_{j} R_{j} \tilde{u}_{j}^{n+1}+2 \sum_{j} \tilde{u}_{j}^{n+1} e_{j}^{n} . \\
& +\kappa
\end{aligned}
$$

Let $\tau$ be an $O(1)$ number to be decided. Combining (3.5) and (3.6), we get

$$
E(n+1)-E(n)+\bar{L}=\bar{R}+\bar{X},
$$


where

$$
\begin{aligned}
& E(n+1)=\sum_{j}\left[\left|\tilde{P}_{j}^{n+1}\right|_{w}^{2}+2 \tau \tilde{u}_{j}^{n+1} W_{j} \tilde{P}_{j}^{n+1}+\tau \frac{\kappa}{1+\kappa}\left|\tilde{u}_{j}^{n+1}\right|_{w}^{2}\right] \\
& E(n)=\sum_{j}\left[\left|\tilde{P}_{j}^{n}\right|_{w}^{2}+2 \tau \tilde{u}_{j}^{n} W_{j} \tilde{P}_{j}^{n}+\tau \frac{\kappa}{1+\kappa}\left|\tilde{u}_{j}^{n}\right|_{w}^{2}\right] \\
& \bar{L}=\frac{2 \kappa}{1+\kappa} \sum_{j}\left|\tilde{P}_{j}^{n}\right|_{w}^{2}+\frac{\mu}{2(1+\kappa)} \sum_{j} \Delta_{+} \tilde{P}_{j}^{n}\left(W_{j}+W_{j+1}\right) \Delta_{+} \tilde{P}_{j}^{n} \\
& +\frac{\kappa \tau}{1+\kappa} \sum_{j} \Delta^{+} \tilde{u}_{j}^{n} W_{j} \Delta^{+} \tilde{u}_{j}^{n}+\frac{\tau \mu^{2}}{2(1+\kappa)} \sum_{j} N_{j}^{n} W_{j} N_{j}^{n} \\
& +\frac{\tau \kappa \mu}{2(1+\kappa)} \sum_{j} M_{j}^{n}\left(W_{j}+W_{j+1}\right) M_{j}^{n} \\
& \bar{X}=\sum_{j}\left|\tilde{P}_{j}^{n+1}-\tilde{P}_{j}^{n}\right|_{w}^{2}-\frac{\mu^{2}}{2(1+\kappa)} \sum_{j} \tilde{P}_{j+1}^{n} \Delta_{0} W_{j} N_{j}^{n} \\
& -\frac{\mu^{2}}{2(1+\kappa)} \sum_{j} \Delta_{0} \tilde{P}_{j}^{n} W_{j-1} N_{j}^{n}+\frac{\mu}{2(1+\kappa)} \sum_{j} \tilde{P}_{j}^{n} \Delta W_{j} \tilde{P}_{j}^{n} \\
& +\frac{\lambda \kappa}{1+\kappa} \sum_{j} \tilde{P}_{j}^{n} W_{j} \Lambda_{j} \Delta_{0} L_{j} R_{j} \tilde{u}_{j}^{n+1}+2 \tau \sum_{j} \Delta^{+} \tilde{u}_{j}^{n} W_{j} \tilde{P}_{j}^{n} \\
& +\frac{\tau \kappa \mu}{1+\kappa} \sum_{j} \Delta^{+} \tilde{u}_{j}^{n} W_{j} L_{j}\left(R_{j+1} \tilde{u}_{j+1}^{n}-2 R_{j} \tilde{u}_{j}^{n}+R_{j-1} \tilde{u}_{j-1}^{n}\right) \\
& +\frac{\tau \kappa \mu}{1+\kappa} \sum_{j} \tilde{u}_{j}^{n} W_{j} L_{j} \Delta_{+} R_{j} \Delta_{+} \tilde{u}_{j}^{n}+\frac{\tau \mu^{2}}{2(1+\kappa)} \sum_{j} \Delta^{+} \tilde{u}_{j}^{n} W_{j} \Delta_{0} N_{j}^{n} \\
& -\frac{\tau \mu^{2}}{2(1+\kappa)} \sum_{j} \tilde{u}_{j+1}^{n} \Delta_{+} W_{j} N_{j}^{n}-\frac{\tau \mu^{2}}{2(1+\kappa)} \sum_{j} \tilde{u}_{j-1}^{n} \Delta_{-} W_{j} N_{j}^{n} \\
& -\frac{\tau \mu}{1+\kappa} \sum_{j} \Delta_{+} \tilde{u}_{j}^{n} W_{j} \Delta_{+} \tilde{P}_{j}^{n}-\frac{\tau \mu}{1+\kappa} \sum_{j} \tilde{u}_{j+1}^{n} \Delta_{+} W_{j} \Delta_{+} \tilde{P}_{j}^{n} \\
& -\frac{\tau \mu}{1+\kappa} \sum_{j} \Delta^{+} \tilde{u}_{j}^{n} W_{j} \Delta \tilde{P}_{j}^{n}+\sum_{j}\left(2 \tilde{P}_{j}^{n}+2 \tau \tilde{u}_{j}^{n+1}\right) W_{j} e_{j}^{n}, \\
& \bar{R}=-\frac{\lambda \kappa}{1+\kappa} \sum_{j}\left(\tilde{P}_{j+1}^{n} W_{j+1}+\tilde{P}_{j}^{n} W_{j}\right) \Lambda_{j+1} \Delta_{+} \tilde{u}_{j}^{n+1} \\
& +\frac{\tau \kappa \mu}{2(1+\kappa)} \sum_{j} \tilde{u}_{j}^{n} \Delta W_{j} \tilde{u}_{j}^{n}+\frac{\tau \kappa \mu}{1+\kappa} \sum_{j} \tilde{u}_{j}^{n} W_{j} L_{j} \Delta R_{j} \tilde{u}_{j}^{n} \\
& -\frac{\tau \kappa \lambda}{1+\kappa} \sum_{j}\left(\tilde{u}_{j+1}^{n+1} W_{j+1}+\tilde{u}_{j}^{n+1} W_{j}\right) \Lambda_{j+1} \Delta_{+} \tilde{u}_{j}^{n+1} \\
& +\frac{\tau \lambda \kappa}{1+\kappa} \sum_{j} \tilde{u}_{j}^{n+1} W_{j} \Lambda_{j} \Delta_{0} L_{j} R_{j} \tilde{u}_{j}^{n+1} .
\end{aligned}
$$

The terms in $\bar{R}$ are labelled as $\bar{R}_{i}$, and the terms in $\bar{X}$ are labelled as $\bar{X}_{i} . \bar{L}$ is positive, so it makes the small perturbation to the discrete shock profile decay to zero. We want to show that $\bar{R}$ produces a negative term of $\left|\tilde{u}_{j}^{n+1}\right|_{w}$ if we choose 
the weight $W$ carefully. We can do that, because the discrete shock profiles have the strong compressibility property. The other terms of $\bar{R}$ and $\bar{X}$ can be estimated by $\bar{L}$ and the positive term of $\left|\tilde{u}_{j}^{n+1}\right|_{w}$ depending on the properties of $W_{j}$ and $\phi_{j}$. For this purpose, some terms in $\bar{R}$ should be changed.

$$
\begin{aligned}
\bar{R}_{1}= & \sum_{j} \frac{\lambda \kappa}{1+\kappa} \tilde{P}_{j}^{n}\left(W_{j+1} \Lambda_{j+1}-W_{j} \Lambda_{j}\right) \tilde{P}_{j}^{n}+\sum_{j} \frac{\lambda \kappa}{1+\kappa} \tilde{P}_{j}^{n} \Delta_{+} W_{j} \Lambda_{j+1} M_{j}^{n+1} \\
& -\sum_{j} \frac{\lambda \kappa}{1+\kappa}\left(\tilde{P}_{j+1}^{n}+\tilde{P}_{j}^{n}\right) W_{j+1} \Lambda_{j+1}\left[(1-\mu) M_{j}^{n}+\frac{\mu}{2}\left(L_{j+1} R_{j+2} \tilde{u}_{j+2}^{n}\right.\right. \\
& \left.\left.-L_{j} R_{j+1} \tilde{u}_{j+1}\right)+\frac{\mu}{2}\left(L_{j+1} R_{j} \tilde{u}_{j}^{n}-L_{j} R_{j-1} \tilde{u}_{j-1}^{n}\right)\right], \\
\bar{R}_{4}= & \frac{\tau \kappa \lambda}{1+\kappa} \sum_{j} \tilde{u}_{j}^{n+1}\left(W_{j+1} \Lambda_{j+1}-W_{j} \Lambda_{j}\right) \tilde{u}_{j}^{n+1} \\
& +\frac{\tau \lambda \kappa}{1+\kappa} \sum_{j} \tilde{u}_{j}^{n+1} \Delta_{+} W_{j} \Lambda_{j+1} \Delta_{+} \tilde{u}_{j}^{n+1} .
\end{aligned}
$$

Because the scheme is implicit, $\tilde{P}_{j}^{n}$ is controlled by $\bar{L}_{1}$ very well, and $\tilde{u}_{j}^{n+1}$ will appear in the transverse terms. We need to change the terms which have $\widetilde{u}_{j}^{n}$ :

$$
\begin{aligned}
\bar{R}_{2}= & \frac{\tau \kappa \mu}{2(1+\kappa)} \sum_{j} \Delta^{+} \tilde{u}_{j}^{n} \Delta W_{j} \Delta^{+} \tilde{u}_{j}^{n}-\frac{\tau \kappa \mu}{1+\kappa} \sum_{j} \Delta^{+} \tilde{u}_{j}^{n} \Delta W_{j} \tilde{u}_{j}^{n+1} \\
& +\frac{\tau \kappa \mu}{2(1+\kappa)} \sum_{j} \tilde{u}_{j}^{n+1} \Delta W_{j} \tilde{u}_{j}^{n+1}, \\
\bar{R}_{3}= & \frac{\tau \kappa \mu}{1+\kappa} \sum_{j} \Delta^{+} \tilde{u}_{j}^{n} W_{j} L_{j} \Delta R_{j} \Delta^{+} \tilde{u}_{j}^{n}-\frac{2 \tau \kappa \mu}{1+\kappa} \sum_{j} \Delta^{+} \tilde{u}_{j}^{n} W_{j} L_{j} \Delta R_{j} \tilde{u}_{j}^{n+1} \\
& +\frac{\tau \kappa \mu}{2(1+\kappa)} \sum_{j} \tilde{u}_{j}^{n+1} W_{j} L_{j} \Delta R_{j} \tilde{u}_{j}^{n+1} .
\end{aligned}
$$

Now, combining the above terms, we have

$$
\begin{aligned}
& \quad \bar{R}=\sum_{j} \frac{\tau \kappa}{1+\kappa} \tilde{u}_{j}^{n+1}\left[\lambda\left(W_{j+1} \Lambda_{j+1}-W_{j} \Lambda_{j}\right)+\lambda W_{j} \Lambda_{j} \Delta_{0} L_{j} R_{j}+\frac{\mu}{2} \Delta W_{j}\right. \\
& \left.+\frac{\mu}{2} W_{j} L_{j} \Delta R_{j}\right] \tilde{u}_{j}^{n+1}+\frac{\tau \lambda \kappa}{1+\kappa} \sum_{j} \tilde{u}_{j}^{n+1} \Delta_{+} W_{j} \Lambda_{j+1} \Delta_{+} \tilde{u}_{j}^{n+1} \\
& +\frac{\tau \kappa \mu}{2(1+\kappa)} \sum_{j} \Delta^{+} \tilde{u}_{j}^{n} \Delta W_{j} \Delta^{+} \tilde{u}_{j}^{n}-\frac{\tau \kappa \mu}{1+\kappa} \sum_{j} \Delta^{+} \tilde{u}_{j}^{n} \Delta W_{j} \tilde{u}_{j}^{n+1} \\
& +\frac{\tau \kappa \mu}{1+\kappa} \sum_{j} \Delta^{+} \tilde{u}_{j}^{n} W_{j} L_{j} \Delta R_{j} \Delta^{+} \tilde{u}_{j}^{n}-\frac{2 \tau \kappa \mu}{1+\kappa} \sum_{j} \Delta^{+} \tilde{u}_{j}^{n} W_{j} L_{j} \Delta R_{j} \tilde{u}_{j}^{n+1} \\
& +\frac{\lambda \kappa}{1+\kappa} \sum_{j} \tilde{P}_{j}^{n}\left(W_{j+1} \Lambda_{j+1}-W_{j} \Lambda_{j}\right) \tilde{P}_{j}^{n}+\sum_{j} \frac{\lambda \kappa}{1+\kappa} \tilde{P}_{j}^{n} \Delta_{+} W_{j} \Lambda_{j+1} M_{j}^{n+1} \\
& -\frac{\lambda \kappa}{1+\kappa} \sum_{j}\left(\tilde{P}_{j+1}^{n}+\tilde{P}_{j}^{n}\right) W_{j+1} \Lambda_{j+1}\left[(1-\mu) M_{j}^{n}+\frac{\mu}{2}\left(L_{j+1} R_{j+2} \tilde{u}_{j+2}^{n}\right.\right. \\
& \left.\left.-L_{j} R_{j+1} \tilde{u}_{j+1}^{n}\right)+\frac{\mu}{2}\left(L_{j+1} R_{j} \tilde{u}_{j}^{n}-L_{j} R_{j-1} \tilde{u}_{j-1}^{n}\right)\right] .
\end{aligned}
$$


Label the above terms as $\bar{R}_{i}$, where $i$ is from 1 to 9 . We want to show that $\bar{R}_{1}$ is negative, so choosing the weights [16]

$$
W_{j}=\operatorname{diag}\left\{w_{1, j}, w_{2, j}, \ldots, w_{m, j}\right\}
$$

as

$$
w_{\mu, j}=\frac{c_{1, \mu}}{\left|\lambda_{\mu, j}\right|} \prod_{i=-\infty}^{j-1}\left(1-c_{2, \mu} \frac{\lambda_{k, i}-\lambda_{k, i+1}}{\lambda_{\mu, i}}\right), \mu \neq \kappa
$$

and

$$
w_{\kappa, j} \equiv 1
$$

we have the following lemma.

Lemma 3.1 (16]). Let $W_{j}$ be the weights defined above. Then we can choose $c_{1, \mu}$ and $c_{2, \mu}$ appropriately so that

$$
\begin{gathered}
W_{j+1}-W_{j}=O(1)\left(\lambda_{k, j}-\lambda_{k, j+1}\right) W_{j}, \\
W_{j+1}-2 W_{j}+W_{j-1}=O(\epsilon)\left(\lambda_{k, j}-\lambda_{k, j+1}\right) W_{j},
\end{gathered}
$$

and

$$
\begin{aligned}
& \lambda\left(W_{j+1} \Lambda_{j+1}-W_{j} \Lambda_{j}\right)+\lambda W_{j} \Lambda_{j}\left(L_{j+1}-L_{j-1}\right) R_{j} \\
& \quad+\frac{\mu}{2}\left(W_{j+1}-2 W_{j}+W_{j-1}\right) \leq-\frac{\lambda}{2}\left(\lambda_{k, j}-\lambda_{k, j+1}\right) W_{j},
\end{aligned}
$$

provided that $\epsilon$ is suitably small.

The position to show is that the transverse terms are controlled by the terms of the principal direction. Using Lemma 3.1, we have these estimates of $\bar{R}_{1}$ and $\bar{R}_{7}$

$$
\begin{aligned}
& \bar{R}_{1} \leq-\frac{\lambda}{2}\left(\lambda_{k, j}-\lambda_{k, j+1}\right) \frac{\tau \kappa}{1+\kappa} \sum_{j}\left|\tilde{u}_{j}^{n+1}\right|_{w}^{2}, \\
& \bar{R}_{7} \leq-\frac{\lambda}{2}\left(\lambda_{k, j}-\lambda_{k, j+1}\right) \frac{\kappa}{1+\kappa} \sum_{j}\left|\tilde{P}_{j}^{n}\right|_{w}^{2} .
\end{aligned}
$$

We only need to estimate $\bar{X}_{1}, \bar{X}_{6}, \bar{X}_{12}, \bar{X}_{15}$, and the other terms can be handled easily. It follows from (3.4) that

$$
\begin{aligned}
& \sum_{j}\left|\tilde{P}_{j}^{n+1}-\tilde{P}_{j}^{n}\right|_{w}^{2} \\
& \leq \frac{5 \kappa}{4(1+\kappa)} \sum_{j}\left|\tilde{P}_{j}^{n}\right|_{w}^{2}+O(1) \frac{\mu^{2}}{(1+\kappa)} \sum_{j}\left|\tilde{P}_{j+1}^{n}-\tilde{P}_{j}^{n}\right|_{w}^{2}+O(1) \frac{\mu^{4}}{1+\kappa} \sum_{j}\left|N_{j}^{n}\right|_{w}^{2} \\
& \quad+O(1) \frac{\kappa \mu^{2}}{1+\kappa} \sum_{j}\left|M_{j}^{n}\right|_{w}^{2}+O(1) \frac{\kappa \mu^{2}}{1+\kappa} \sum_{j}\left|\tilde{u}_{j}^{n+1}-\tilde{u}_{j}^{n}\right|_{w}^{2} \\
& \quad+O(\epsilon) \sum_{j}\left(\lambda_{k, j}-\lambda_{k, j+1}\right)\left|\tilde{u}_{j}^{n+1}\right|_{w}^{2}+O(1) \sum_{j}\left|e_{j}^{n}\right|_{w}^{2} .
\end{aligned}
$$

Set

$$
\bar{M}\left(n_{1}\right)=\sup _{n \leq n_{1}}\left(\sum_{j}\left|\tilde{u}_{j}^{n}\right|^{2}+\sum_{j}\left|\tilde{P}_{j}^{n}\right|^{2}\right)^{\frac{1}{2}}
$$


and assume that $\bar{M}\left(n_{1}\right)$ is small enough. Clearly, we have

$$
\sup _{n \leq n_{1}, j}\left(\left|\tilde{u}_{j}^{n}\right|+\left|\tilde{P}_{j}^{n}\right|\right) \leq \bar{M}\left(n_{1}\right)
$$

and because

$$
\left|\theta_{j}^{n+1}\right| \leq O(1)\left(\left|\tilde{u}_{j}^{n+1}-\tilde{u}_{j+1}^{n+1}\right|^{2}+\left(\lambda_{k, j}-\lambda_{k, j+1}\right)^{2}\left|\tilde{u}_{j}^{n+1}\right|^{2}\right),
$$

immediately, by Theorem 2.3 , we have

$$
\begin{aligned}
\sum_{j}\left|e_{j}^{n}\right|_{w}^{2} \leq & O(1) \sum_{j} \frac{\kappa \mu^{2}}{1+\kappa}\left|\left(\lambda_{k, j}-\lambda_{k, j+1}\right) \tilde{u}_{j}^{n+1}\right|_{w}^{2}+O(\epsilon) \sum_{j} \frac{\kappa \mu^{4}}{1+\kappa}\left|M_{j}^{n}\right|_{w}^{2} \\
& +O(1) \sum_{j} \frac{\kappa \mu^{2}}{1+\kappa}\left|\left(\lambda_{k, j}-\lambda_{k, j+1}\right) M_{j}^{n+1}\right|_{w}^{2} \\
& +O(1) \sum_{j} \frac{\mu^{2}}{1+\kappa}\left|\left(\lambda_{k, j}-\lambda_{k, j+1}\right)\left(\tilde{P}_{j+1}^{n}-\tilde{P}_{j}^{n}\right)\right|_{w}^{2} \\
& +O(1) \sum_{j} \frac{\mu^{2}}{1+\kappa}\left|\left(\lambda_{k, j}-\lambda_{k, j+1}\right) \tilde{P}_{j}^{n}\right|_{w}^{2} \\
& +O(\epsilon) \sum_{j} \frac{\mu^{4}}{1+\kappa}\left|\Delta^{+} \tilde{u}_{j}^{n}\right|_{w}^{2}+\sum_{j} O(1) \bar{M}\left(n_{1}\right)^{2} \frac{\kappa \mu^{2}}{1+\kappa}\left|M_{j}^{n+1}\right|_{w}^{2}
\end{aligned}
$$

so

$$
\begin{aligned}
& \sum_{j}\left|\tilde{P}_{j}^{n+1}-\tilde{P}_{j}^{n}\right|_{w}^{2} \\
& \leq \frac{5 \kappa}{4(1+\kappa)} \sum_{j}\left|\tilde{P}_{j}^{n}\right|_{w}^{2}+O(1) \frac{\kappa \mu^{2}}{1+\kappa} \sum_{j}\left|\Delta^{+} \tilde{u}_{j}^{n}\right|_{w}^{2} \\
& \quad+O(1) \sum_{j} \frac{\kappa \mu^{2}}{1+\kappa}\left|M_{j}^{n}\right|_{w}^{2}+\frac{\lambda}{8} \sum_{j}\left(\lambda_{k, j}-\lambda_{k, j+1}\right)\left|\tilde{u}_{j}^{n+1}\right|_{w}^{2} \\
& \quad+O(1) \frac{\mu^{2}}{1+\kappa} \sum_{j}\left|\tilde{P}_{j+1}^{n}-\tilde{P}_{j}^{n}\right|_{w}^{2}+O(\epsilon) \sum_{j} \frac{\mu^{2}}{1+\kappa}\left|\tilde{P}_{j}^{n}\right|_{w}^{2} \\
& \quad+O(1) \sum_{j} \bar{M}\left(n_{1}\right)^{2} \frac{\kappa \mu^{2}}{1+\kappa}\left|M_{j}^{n+1}\right|_{w}^{2} .
\end{aligned}
$$

$\bar{X}_{6}$ and $\bar{X}_{12}$ should be dealt with more carefully. We can estimate these terms as

$$
\begin{aligned}
\bar{X}_{6} \leq & \left(2 \tau+O(1) \tau \mu+O(1) \tau \mu^{\frac{1}{2}}\right) \sum_{j}\left|\tilde{P}_{j}^{n}\right|_{w}^{2}+\frac{\tau \kappa \mu}{4(1+\kappa)} \sum_{j}\left|M_{j}^{n}\right|_{w}^{2} \\
& +\frac{\mu^{\frac{3}{2}}}{2} \sum_{j}\left(\lambda_{k, j}-\lambda_{k, j+1}\right)\left|\tilde{u}_{j}^{n+1}\right|_{w}^{2}+\frac{\mu}{2} \sum_{j}\left(\lambda_{k, j}-\lambda_{k, j+1}\right)\left|\Delta^{+} \tilde{u}_{j}^{n}\right|_{w}^{2}, \\
\bar{X}_{12} \leq & \frac{\mu}{1+\kappa}\left[\frac{1}{2} \sum_{j} \tilde{P}_{j}^{n} W_{j} \tilde{P}_{j}^{n}+\frac{\tau^{2}}{2} \sum_{j}\left|M_{j}^{n}\right|_{w}^{2}\right] .
\end{aligned}
$$


By Lemma 3.1 we have

$$
\begin{aligned}
\bar{X}_{15} \leq & \frac{\lambda}{8} \sum_{j}\left(\lambda_{k, j}-\lambda_{k, j+1}\right)\left|\tilde{u}_{j}^{n+1}\right|_{w}^{2}+\frac{\lambda}{8} \sum_{j}\left(\lambda_{k, j}-\lambda_{k, j+1}\right)\left|\tilde{P}_{j}^{n}\right|_{w}^{2} \\
& +\frac{\lambda \kappa}{1+\kappa}\left(O(\epsilon)+\bar{M}\left(n_{1}\right)\right) \sum_{j}\left(\left|M_{j}^{n}\right|_{w}^{2}+\left|\tilde{u}_{j}^{n+1}-\tilde{u}_{j}^{n}\right|_{w}^{2}\right) \\
& +O(1) \frac{\mu^{2}}{1+\kappa} \sum_{j}\left|\tilde{u}_{j+1}^{n}-\tilde{u}_{j}^{n}\right|_{w}^{2}+O(\epsilon) \frac{\mu}{1+\kappa} \sum_{j}\left|\tilde{P}_{j+1}^{n}-\tilde{P}_{j}^{n}\right|_{w}^{2} .
\end{aligned}
$$

Two typical terms can be estimated as follows:

$$
\begin{aligned}
& \sum_{j}\left|\tilde{u}_{j}^{n+1} W_{j} L_{j}\left(R_{j+1}-R_{j}\right) \Lambda_{j+1}\left(\tilde{u}_{j+1}^{n+1}-\tilde{u}_{j}^{n+1}\right)\right| \\
& \quad \leq O(1) \sum_{j}\left(\lambda_{k, j}-\lambda_{k, j+1}\right)\left|\tilde{u}_{j}^{n+1} \cdot W_{j}\left(\tilde{u}_{j+1}^{n+1}-\tilde{u}_{j}^{n+1}\right)\right| \\
& \quad \leq \sum_{j} \frac{1}{48}\left(\lambda_{k, j}-\lambda_{k, j+1}\right)\left|\tilde{u}_{j}^{n+1}\right|_{w}^{2}+\sum_{j} O(\epsilon)\left|\tilde{u}_{j+1}^{n+1}-\tilde{u}_{j}^{n+1}\right|_{w}^{2}, \\
& \sum_{j}\left|\tilde{u}_{j}^{n+1} \cdot W_{j} L_{j} \theta_{j}^{n+1}\right| \\
& \quad \leq O(1) \sum_{j}\left|W_{j} \tilde{u}_{j}^{n+1}\right|\left|\tilde{u}_{j}^{n+1}-\tilde{u}_{j+1}^{n+1}\right|^{2}+\sum_{j} O(\epsilon)\left(\lambda_{k, j}-\lambda_{k, j+1}\right)\left|\tilde{u}_{j}^{n+1}\right|_{w}^{2} \\
& \quad \leq O(1) \bar{M}\left(n_{1}\right) \sum_{j}\left|\tilde{u}_{j}^{n+1}-\tilde{u}_{j+1}^{n+1}\right|_{w}^{2}+O(\epsilon) \sum_{j}\left(\lambda_{k, j}-\lambda_{k, j+1}\right)\left|\tilde{u}_{j}^{n+1}\right|_{w}^{2} .
\end{aligned}
$$

Set $\tau=\frac{\kappa}{4(1+\kappa)}$. If $\epsilon$ and $\lambda$ are suitably small, we will obtain

$$
\begin{gathered}
E(n+1)-E(n)+\frac{\lambda}{4} \sum_{j}\left(\lambda_{k, j}-\lambda_{k, j+1}\right)\left[\left|\tilde{u}_{j}^{n+1}\right|_{w}^{2}+\left|\tilde{P}_{j}^{n}\right|_{w}^{2}\right]+O(1) \sum_{j}\left|\tilde{P}_{j}^{n}\right|_{w}^{2} \\
+O(1)\left[\sum_{j}\left|\tilde{u}_{j}^{n}-\tilde{u}_{j+1}^{n}\right|_{w}^{2}+\sum_{j}\left|\tilde{P}_{j}^{n}-\tilde{P}_{j+1}^{n}\right|_{w}^{2}+\sum_{j}\left|\tilde{u}_{j}^{n+1}-\tilde{u}_{j}^{n}\right|_{w}^{2}\right] \leq 0 .
\end{gathered}
$$

Since the weights are bounded by some positive constants, if $\epsilon$ and $\lambda$ are suitably small, we have the basic a priori estimate.

Proposition 3.2 (A priori estimate). Let $\left(\tilde{u}^{n}, \tilde{P}^{n}\right)$ be a solution of (3.4) for $n \leq$ $n_{1}$. Then there exists a positive constant $C$ independent of $n_{1}$ and $\epsilon$ such that for $n_{2} \leq n_{1}$

$$
\begin{aligned}
& \sum_{j}\left(\left|\tilde{u}_{j}^{n_{2}}\right|^{2}+\left|\tilde{P}_{j}^{n_{2}}\right|^{2}\right)+\sum_{n \leq n_{2}} \sum_{j}\left|\lambda_{k, j}-\lambda_{k, j+1}\right|\left[\left|\tilde{u}_{j}^{n}\right|^{2}+\left|\tilde{P}_{j}^{n}\right|^{2}\right] \\
& \quad+\sum_{n \leq n_{2}} \sum_{j}\left[\left|\tilde{P}_{j}^{n}\right|^{2}+\left|\tilde{P}_{j}^{n}-\tilde{P}_{j+1}^{n}\right|^{2}+\left|\tilde{u}_{j}^{n}-\tilde{u}_{j+1}^{n}\right|^{2}\right] \leq C \sum_{j}\left[\left|\tilde{u}_{j}^{0}\right|^{2}+\left|\tilde{P}_{j}^{0}\right|^{2}\right],
\end{aligned}
$$

provided that $\epsilon, \lambda$ and $\bar{M}\left(n_{1}\right)$ are suitably small.

In fact, if the initial data is small enough, i.e., $\bar{M}(0)$ is small enough, then (B.9) is true a little longer and hence forever by the standard continuity argument. Then the following proposition holds. 
Proposition 3.3. Assume that $\epsilon$ and $\bar{M}(0)$ are suitably small. Then the problem (3.4) has a unique global solution $\left(\tilde{u}_{j}^{n}, \tilde{P}_{j}^{n}\right)$. For any $n \geq 0$,

$$
\begin{aligned}
& \sup _{n} \sum_{j}\left(\left|\tilde{u}_{j}^{n}\right|^{2}+\left|\tilde{P}_{j}^{n}\right|^{2}\right)+\sum_{j, n}\left|\lambda_{k, j}-\lambda_{k, j+1}\right|\left[\left|\tilde{u}_{j}^{n}\right|^{2}+\left|\tilde{P}_{j}^{n}\right|^{2}\right] \\
& +\sum_{j, n}\left[\left|\tilde{P}_{j}^{n}\right|^{2}+\left|\tilde{P}_{j}^{n}-\tilde{P}_{j+1}^{n}\right|^{2}+\left|\tilde{u}_{j}^{n}-\tilde{u}_{j+1}^{n}\right|^{2}\right] \leq C \bar{M}(0)^{2},
\end{aligned}
$$

where $C$ is a positive constant independent of $n$ and $j$.

Now it remains to estimate the $\bar{v}_{j}^{n}$, because

$$
\begin{aligned}
(1+\kappa) \bar{v}_{j}^{n+1}= & \bar{v}_{j}^{n}+A^{\frac{1}{2}} \lambda\left(\bar{v}_{j+1}^{n}-2 \bar{v}_{j}^{n}+\bar{v}_{j-1}^{n}\right)-\frac{\lambda A}{2}\left(\bar{u}_{j+1}^{n}-\bar{u}_{j-1}^{n}\right) \\
& +\kappa\left(\nabla F\left(\phi_{j}\right) \bar{u}_{j}^{n+1}\right)+\kappa O(1)\left|\bar{u}_{j}^{n+1}\right|^{2} .
\end{aligned}
$$

Multiply the above equation by $\bar{v}_{j}^{n+1}$, and perform summation by parts. Immediately, we have the estimate

$$
\sum_{j}\left|\bar{v}_{j}^{n+1}\right|^{2}-\nu \sum_{j}\left|\bar{v}_{j}^{n}\right|^{2}+O(1) \sum_{j}\left|\bar{v}_{j}^{n}-\bar{v}_{j+1}^{n}\right|^{2} \leq C \sum_{j}\left|\hat{u}_{j}^{n}-\hat{u}_{j+1}^{n}\right|^{2}
$$

provided that $\lambda$ and $\bar{M}(0)$ are suitably small, where $\nu<1$. Note that $O(1)$ and $C$ are independent of $n$. Combining this with (3.10), we have the global estimate

$$
\sup _{n} \sum_{j}\left|\bar{v}_{j}^{n}\right|^{2}+O(1) \sum_{j, n}\left|\bar{v}_{j}^{n}-\bar{v}_{j+1}^{n}\right|^{2} \leq C \bar{M}^{2}(0),
$$

from above. Under the assumption of initial conditions, similarly to [16], we have

$$
\lim _{n \rightarrow+\infty}\left[\sum_{j}\left|u_{j}^{n}-\phi_{j}\right|^{2}+\left|v_{j}^{n}-\psi_{j}\right|^{2}\right]=0 .
$$

This is our Theorem 1.1

\section{4. $L^{2}$ STABILITY OF NONSTATIONARY DisCRETE SHOCK PROFILES}

In this section we will prove the $L^{2}$ stability of the single discrete shock profile. Here we assume the discrete shock speed $s>0$ (the case of $s<0$ can be handled by a little modification). Because of the complex structure of the nonstationary discrete shock profile, the method discussed in the last section cannot be used, so we use the idea of vertical estimate. As before, let $\left(\phi_{j}^{n}, \psi_{j}^{n}\right)$ be the single discrete shock profile in the $k$-field of the equilibrium system as follows:

$$
\begin{gathered}
\phi_{j}^{n+1}-\phi_{j}^{n}+\frac{k}{2 h}\left(\psi_{j+1}^{n}-\psi_{j-1}^{n}\right)-\frac{k}{2 h} A^{\frac{1}{2}}\left(\phi_{j+1}^{n}-2 \phi_{j}^{n}+\phi_{j-1}^{n}\right)=0, \\
\psi_{j}^{n+1}-\psi_{j}^{n}+\frac{k}{2 h} A\left(\phi_{j+1}^{n}-\phi_{j-1}^{n}\right)-\frac{k}{2 h} A^{\frac{1}{2}}\left(\psi_{j+1}^{n}-2 \psi_{j}^{n}+\psi_{j-1}^{n}\right) \\
=\kappa\left(F\left(\phi_{j}^{n+1}\right)-\psi_{j}^{n+1}\right) .
\end{gathered}
$$

The definition of $\bar{u}_{j}^{n}, P_{j}^{n}, \hat{P}_{j}^{n}$ is the same as in the stationary case. Also let

$$
P_{j}^{n}=R_{j}^{n} \tilde{P}_{j}^{n}, \hat{u}_{j}^{n}=R_{j}^{n} \tilde{u}_{j}^{n} .
$$


Immediately, we have

$$
\begin{aligned}
\tilde{P}_{j}^{n+1} & -\tilde{P}_{j}^{n}+\frac{\kappa}{1+\kappa} \tilde{P}_{j}^{n}-\frac{\mu^{2}}{4(1+\kappa)}\left(\tilde{u}_{j+2}^{n}-2 \tilde{u}_{j}^{n}+\tilde{u}_{j-2}^{n}\right) \\
& -\frac{\mu}{2(1+\kappa)}\left(\tilde{P}_{j+1}^{n}-2 \tilde{P}_{j}^{n}+\tilde{P}_{j-1}^{n}\right) \\
& +\frac{\lambda \kappa}{2(1+\kappa)} \Lambda_{j}^{n+1}\left(\tilde{u}_{j}^{n+1}-\tilde{u}_{j-1}^{n+1}\right) \\
& +\frac{\lambda \kappa}{2(1+\kappa)} \Lambda_{j+1}^{n+1}\left(\tilde{u}_{j+1}^{n+1}-\tilde{u}_{j}^{n+1}\right) \\
& =\frac{1}{1+\kappa}\left(L_{j}^{n+1}-L_{j}^{n}\right) R_{j}^{n} \tilde{P}_{j}^{n} \\
& +\frac{\lambda \kappa}{2(1+\kappa)} \Lambda_{j}^{n+1}\left(L_{j+1}^{n+1}-L_{j-1}^{n+1}\right) R_{j}^{n+1} \tilde{u}_{j}^{n+1}+e_{j}^{n},
\end{aligned}
$$

where

$$
\begin{aligned}
e_{j}^{n}= & \frac{\lambda \kappa}{2(1+\kappa)} \Delta_{+} \Lambda_{j}^{n+1} \Delta_{+} L_{j}^{n+1} R_{j}^{n+1} \tilde{u}_{j}^{n+1} \\
& +\frac{\mu}{2(1+\kappa)} L_{j}^{n} \Delta_{+} R_{j}^{n} \tilde{P}_{j+1}^{n} \\
& -\frac{\lambda \kappa}{2(1+\kappa)} L_{j}^{n+1} \Delta_{+} R_{j}^{n+1} \Lambda_{j+1}^{n+1} \Delta_{+} \tilde{u}_{j}^{n+1} \\
& +\frac{\lambda \kappa}{2(1+\kappa)} L_{j}^{n+1} \Delta_{+} R_{j}^{n+1} \Lambda_{j+1}^{n+1} \Delta_{+} L_{j}^{n+1} R_{j}^{n+1} \tilde{u}_{j}^{n+1} \\
& -\frac{\lambda \kappa}{2(1+\kappa)} \Lambda_{j}^{n+1} \Delta_{-} L_{j}^{n+1} R_{j-1}^{n+1} \Delta_{-} \tilde{u}_{j}^{n+1} \\
& -\frac{\lambda \kappa}{2(1+\kappa)} \Lambda_{j}^{n+1} \Delta_{-} L_{j}^{n+1} \Delta_{-} R_{j}^{n+1} \tilde{u}_{j}^{n+1} \\
& +\frac{\mu^{2}}{4(1+\kappa)} \Delta^{+} L_{j}^{n}\left(R_{j+2}^{n} \tilde{u}_{j+2}^{n}-2 R_{j}^{n} \tilde{u}_{j}^{n}+R_{j-2}^{n} \tilde{u}_{j-2}^{n}\right) \\
& +\frac{\mu^{2}}{4(1+\kappa)} L_{j}^{n}\left(R_{j+2}^{n}-R_{j}^{n}\right) \tilde{u}_{j+2}^{n} \\
& +\frac{\mu^{2}}{4(1+\kappa)} L_{j}^{n}\left(R_{j-2}^{n}-R_{j}^{n}\right) \tilde{u}_{j-2}^{n} \\
& +\frac{\mu}{2(1+\kappa)} \Delta^{+} L_{j}^{n}\left(R_{j+1}^{n} \tilde{P}_{j+1}^{n}-2 R_{j}^{n} \tilde{P}_{j}^{n}+R_{j-1}^{n} \tilde{P}_{j-1}^{n}\right) \\
& -\frac{\mu}{2(1+\kappa)} L_{j}^{n} \Delta_{-} R_{j}^{n} \tilde{P}_{j-1}^{n} \\
& -\frac{\kappa \lambda}{2(1+\kappa)} L_{j}^{n+1}\left[\theta_{j}^{n+1}+\theta_{j+1}^{n+1}\right] . \\
&
\end{aligned}
$$

Note that

$$
P_{j}^{n}=R_{j}^{n+1} \tilde{u}_{j}^{n+1}-R_{j}^{n} \tilde{u}_{j}^{n}-\frac{\lambda}{2} A^{\frac{1}{2}}\left(R_{j+1}^{n} \tilde{u}_{j+1}^{n}-2 R_{j}^{n} \tilde{u}_{j}^{n}+R_{j-1}^{n} \tilde{u}_{j-1}^{n}\right) .
$$


Next, assume $2 \tilde{P}_{j}^{n} \times(4.2)$. Then performing summation by parts leads to

$$
\begin{aligned}
\sum_{j}\left|\tilde{P}_{j}^{n+1}\right|^{2}-\sum_{j}\left|\tilde{P}_{j}^{n}\right|^{2}-\sum_{j}\left|\tilde{P}_{j}^{n+1}-\tilde{P}_{j}^{n}\right|^{2}+\frac{2 \kappa}{1+\kappa} \sum_{j}\left|\tilde{P}_{j}^{n}\right|^{2} \\
\quad+\frac{\mu}{1+\kappa} \sum_{j}\left|\tilde{P}_{j+1}^{n}-\tilde{P}_{j}^{n}\right|^{2} \\
\quad+\sum_{j} \frac{\mu^{2}}{2(1+\kappa)}\left(\tilde{P}_{j+1}^{n}-\tilde{P}_{j-1}^{n}\right) N_{j}^{n} \\
\quad+\sum_{j} \frac{\lambda \kappa}{1+\kappa} \tilde{P}_{j}^{n}\left(\Lambda_{j}^{n+1}-\Lambda_{j+1}^{n+1}\right) \tilde{P}_{j}^{n} \\
\quad+\frac{\lambda \kappa}{1+\kappa} \sum_{j}\left(\tilde{P}_{j+1}^{n}+\tilde{P}_{j}^{n}\right) \Lambda_{j+1}^{n+1}\left[\Delta_{0} L_{j}^{n+1} \tilde{P}_{j+1}^{n}-\Delta^{+} L_{j}^{n} \tilde{P}_{j}^{n}\right] \\
\quad+\frac{\lambda \kappa}{1+\kappa}(1-\mu) \sum_{j}\left(\tilde{P}_{j+1}^{n}+\tilde{P}_{j}^{n}\right) \Lambda_{j+1}^{n+1}\left(L_{j}^{n+1} R_{j}^{n} \tilde{u}_{j}^{n}-L_{j+1}^{n+1} R_{j+1}^{n} \tilde{u}_{j+1}^{n}\right) \\
\quad+\frac{\lambda \kappa \mu}{2(1+\kappa)} \sum_{j}\left(\tilde{P}_{j+1}^{n}+\tilde{P}_{j}^{n}\right) \Lambda_{j+1}^{n+1}\left(L_{j}^{n+1} R_{j+1}^{n} \tilde{u}_{j+1}^{n}-L_{j+1}^{n+1} R_{j+2}^{n} \tilde{u}_{j+2}^{n}\right) \\
\quad+\frac{\lambda \kappa \mu}{2(1+\kappa)} \sum_{j}\left(\tilde{P}_{j+1}^{n}+\tilde{P}_{j}^{n}\right) \Lambda_{j+1}^{n+1}\left(L_{j}^{n+1} R_{j-1}^{n} \tilde{u}_{j-1}^{n}-L_{j+1}^{n+1} R_{j}^{n} \tilde{u}_{j}^{n}\right) \\
=\sum_{j} \frac{2}{1+\kappa} \tilde{P}_{j}^{n} \Delta^{+} L_{j}^{n} R_{j}^{n} \tilde{P}_{j}^{n} \\
\quad+\sum_{j} \frac{\lambda \kappa}{1+\kappa} \tilde{P}_{j}^{n} \Lambda_{j}^{n+1} \Delta_{0} L_{j}^{n+1} R_{j}^{n+1} \tilde{u}_{j}^{n+1} \\
+\sum_{j} 2 \tilde{P}_{j}^{n} e_{j}^{n} . \\
\end{aligned}
$$

The key part to deriving the above equation is

$$
I=\sum_{j} \frac{\lambda \kappa}{1+\kappa}\left(\tilde{P}_{j+1}^{n} \Lambda_{j+1}^{n+1}+\tilde{P}_{j}^{n} \Lambda_{j+1}^{n+1}\right)\left(\tilde{u}_{j+1}^{n+1}-\tilde{u}_{j}^{n+1}\right) .
$$

Because

$$
\begin{aligned}
\tilde{u}_{j+1}^{n+1}-\tilde{u}_{j}^{n+1}= & \tilde{P}_{j+1}^{n}-\tilde{P}_{j}^{n}+\Delta^{+} L_{j+1}^{n} \tilde{P}_{j+1}^{n}-\Delta^{+} L_{j}^{n} \tilde{P}_{j}^{n} \\
& +\frac{\mu}{2}\left(L_{j}^{n+1} R_{j+1}^{n} \tilde{u}_{j+1}^{n}-L_{j+1}^{n+1} R_{j+2}^{n} \tilde{u}_{j+2}^{n}\right) \\
& +\left(L_{j}^{n+1} R_{j}^{n} \tilde{u}_{j}^{n}-L_{j+1}^{n+1} R_{j+1}^{n} \tilde{u}_{j+1}^{n}\right)(1-\mu) \\
& +\frac{\mu}{2}\left(L_{j}^{n+1} R_{j-1}^{n} \tilde{u}_{j-1}^{n}-L_{j+1}^{n+1} R_{j}^{n} \tilde{u}_{j}^{n}\right),
\end{aligned}
$$


Then

$$
\begin{aligned}
I= & \sum_{j} \frac{\lambda \kappa}{1+\kappa}\left(\tilde{P}_{j+1}^{n}+\tilde{P}_{j}^{n}\right) \Lambda_{j+1}^{n+1} \Delta_{+} \tilde{P}_{j}^{n} \\
& +\sum_{j} \frac{\lambda \kappa}{1+\kappa}\left(\tilde{P}_{j+1}^{n}+\tilde{P}_{j}^{n}\right) \Lambda_{j+1}^{n+1}\left[\Delta^{+} L_{j+1}^{n} \tilde{P}_{j+1}^{n}-\Delta^{+} L_{j}^{n} \tilde{P}_{j}^{n}\right] \\
& +\sum_{j} \frac{\lambda \kappa}{1+\kappa}\left(\tilde{P}_{j+1}^{n}+\tilde{P}_{j}^{n}\right) \Lambda_{j+1}^{n+1}\left(L_{j}^{n+1} R_{j}^{n} \tilde{u}_{j}^{n}-L_{j+1}^{n+1} R_{j+1}^{n} \tilde{u}_{j+1}^{n}\right)(1-\mu) \\
& +\sum_{j} \frac{\lambda \kappa}{1+\kappa}\left(\tilde{P}_{j+1}^{n}+\tilde{P}_{j}^{n}\right) \Lambda_{j+1}^{n+1}\left(L_{j}^{n+1} R_{j+1}^{n} \tilde{u}_{j+1}^{n}-L_{j+1}^{n+1} R_{j+2}^{n} \tilde{u}_{j+2}^{n}\right) \frac{\mu}{2} \\
& +\sum_{j} \frac{\lambda \kappa}{1+\kappa}\left(\tilde{P}_{j+1}^{n}+\tilde{P}_{j}^{n}\right) \Lambda_{j+1}^{n+1}\left(L_{j}^{n+1} R_{j-1}^{n} \tilde{u}_{j-1}^{n}-L_{j+1}^{n+1} R_{j}^{n} \tilde{u}_{j}^{n}\right) \frac{\mu}{2} .
\end{aligned}
$$

Next, $2 \tilde{u}_{j}^{n+1} \times 4.2$ can be rewritten using summation by parts:

$$
\begin{aligned}
& 2 \sum_{j} \tilde{u}_{j}^{n+1} \tilde{P}_{j}^{n+1}-2 \sum_{j} \tilde{u}_{j}^{n} \tilde{P}_{j}^{n}+2 \sum_{j}\left(\tilde{u}_{j}^{n}-\tilde{u}_{j}^{n+1}\right) \tilde{P}_{j}^{n} \\
& +\sum_{j} \frac{\kappa}{1+\kappa}\left|\tilde{u}_{j}^{n+1}\right|^{2}-\sum_{j} \frac{\kappa}{1+\kappa}\left|\tilde{u}_{j}^{n}\right|^{2} \\
& +\sum_{j} \frac{\kappa}{1+\kappa}\left|\Delta^{+} \tilde{u}_{j}^{n}\right|^{2}-\sum_{j} \frac{2 \kappa}{1+\kappa} \tilde{u}_{j}^{n+1} \Delta^{+} L_{j}^{n} R_{j}^{n+1} \tilde{u}_{j}^{n+1} \\
& -\frac{\kappa \mu}{1+\kappa} \sum_{j} \Delta^{+} \tilde{u}_{j}^{n} L_{j}^{n}\left(R_{j+1}^{n} \tilde{u}_{j+1}^{n}-2 R_{j}^{n} \tilde{u}_{j}^{n}+R_{j-1}^{n} \tilde{u}_{j-1}^{n}\right) \\
& -\frac{\kappa \mu}{1+\kappa} \sum_{j} \tilde{u}_{j}^{n} L_{j}^{n} \Delta_{+} R_{j}^{n} \Delta_{+} \tilde{u}_{j}^{n} \\
& -\frac{\kappa \mu}{1+\kappa} \sum_{j} \tilde{u}_{j}^{n} L_{j}^{n} \Delta_{-} R_{j}^{n} \Delta_{-} \tilde{u}_{j}^{n} \\
& -\frac{\kappa \mu}{1+\kappa} \sum_{j} \tilde{u}_{j}^{n} L_{j}^{n} \Delta R_{j}^{n} \tilde{u}_{j}^{n} \\
& +\frac{\kappa \mu}{1+\kappa} \sum_{j}\left|\tilde{u}_{j+1}^{n}-\tilde{u}_{j}^{n}\right|^{2} \\
& -\sum_{j} \frac{\mu^{2}}{2(1+\kappa)} \Delta^{+} \tilde{u}_{j}^{n}\left(N_{j+1}^{n}-N_{j-1}^{n}\right)+\frac{\mu^{2}}{2(1+\kappa)} \sum_{j}\left|N_{j}^{n}\right|^{2} \\
& -\sum_{j} \frac{\mu}{1+\kappa} \Delta^{+} \tilde{u}_{j}^{n} \Delta \tilde{P}_{j}^{n}+\sum_{j} \frac{\mu}{1+\kappa} \Delta_{+} \tilde{u}_{j}^{n} \Delta_{+} \tilde{P}_{j}^{n} \\
& -\sum_{j} \frac{\lambda \kappa}{1+\kappa} \tilde{u}_{j}^{n+1} \Delta_{+} \Lambda_{j}^{n+1} \tilde{u}_{j}^{n+1}-\sum_{j} \frac{2}{1+\kappa} \tilde{u}_{j}^{n+1} \Delta^{+} L_{j}^{n} R_{j}^{n} \tilde{P}_{j}^{n} \\
& =\frac{\lambda \kappa}{1+\kappa} \sum_{j} \tilde{u}_{j}^{n+1} \Lambda_{j}^{n+1} \Delta_{0} L_{j}^{n+1} R_{j}^{n+1} \tilde{u}_{j}^{n+1}+\sum_{j} 2 \tilde{u}_{j}^{n+1} e_{j}^{n} .
\end{aligned}
$$


The only term which we should be careful of is

$$
\begin{aligned}
J \equiv & \sum_{j} \frac{2 \kappa}{1+\kappa} \tilde{P}_{j}^{n} \tilde{u}_{j}^{n+1} \\
= & \sum_{j} \frac{2 \kappa}{1+\kappa} \tilde{u}_{j}^{n+1}\left(L_{j}^{n} R_{j}^{n+1} \tilde{u}_{j}^{n+1}-\tilde{u}_{j}^{n}\right. \\
& \left.-\frac{\mu}{2} L_{j}^{n}\left(R_{j+1}^{n} \tilde{u}_{j+1}^{n}-2 R_{j}^{n} \tilde{u}_{j}^{n}+R_{j-1}^{n} \tilde{u}_{j-1}^{n}\right)\right) \\
= & \sum_{j} \frac{\kappa}{1+\kappa}\left|\tilde{u}_{j}^{n+1}\right|^{2}-\sum_{j} \frac{\kappa}{1+\kappa}\left|\tilde{u}_{j}^{n}\right|^{2}+\sum_{j} \frac{\kappa}{1+\kappa}\left|\Delta^{+} \tilde{u}_{j}^{n}\right|^{2} \\
& -\sum_{j} \frac{2 \kappa}{1+\kappa} \tilde{u}_{j}^{n+1} \Delta^{+} L_{j}^{n} R_{j}^{n+1} \tilde{u}_{j}^{n+1} \\
& -\frac{\kappa \mu}{1+\kappa} \sum_{j} \tilde{u}_{j}^{n} L_{j}^{n} \Delta_{+} R_{j}^{n} \Delta_{+} \tilde{u}_{j}^{n} \\
& -\frac{\kappa \mu}{1+\kappa} \sum_{j} \Delta^{+} \tilde{u}_{j}^{n} L_{j}^{n}\left(R_{j+1}^{n} \tilde{u}_{j+1}^{n}-2 R_{j}^{n} \tilde{u}_{j}^{n}+R_{j-1}^{n} \tilde{u}_{j-1}^{n}\right) \\
& +\frac{\kappa \mu}{1+\kappa} \sum_{j}\left|\Delta_{+} \tilde{u}_{j}^{n}\right|^{2}-\frac{\kappa \mu}{1+\kappa} \sum_{j} \tilde{u}_{j}^{n} L_{j}^{n} \Delta_{-} R_{j}^{n} \Delta_{-} \tilde{u}_{j}^{n} \\
& -\frac{\kappa \mu}{1+\kappa} \sum_{j} \tilde{u}_{j}^{n} L_{j}^{n} \Delta R_{j}^{n} \tilde{u}_{j}^{n} .
\end{aligned}
$$

It follows from (4.5) $+\tau \times(4.6)$ that

$$
E(n+1)-E(n)+L(n)=R(n),
$$

where

$$
\begin{aligned}
E(n+1) & =\sum_{j}\left[\left|\tilde{P}_{j}^{n+1}\right|^{2}+2 \tau \tilde{u}_{j}^{n+1} \tilde{P}_{j}^{n+1}+\frac{\kappa \tau}{1+\kappa}\left|\tilde{u}_{j}^{n+1}\right|^{2}\right] \\
E(n)= & \sum_{j}\left[\left|\tilde{P}_{j}^{n}\right|^{2}+2 \tau \tilde{u}_{j}^{n} \tilde{P}_{j}^{n}+\frac{\kappa \tau}{1+\kappa}\left|\tilde{u}_{j}^{n}\right|^{2}\right] \\
L(n)= & \frac{2 \kappa}{1+\kappa} \sum_{j}\left|\tilde{P}_{j}^{n}\right|^{2}+\frac{\mu}{1+\kappa} \sum_{j}\left|\tilde{P}_{j+1}^{n}-\tilde{P}_{j}^{n}\right|^{2} \\
& +\frac{\lambda \kappa}{1+\kappa} \sum_{j}\left(\lambda_{k, j}^{n+1}-\lambda_{k, j+1}^{n+1}\right)\left|\tilde{P}_{k, j}^{n}\right|^{2} \\
& +\frac{\tau \kappa}{1+\kappa} \sum_{j}\left|\tilde{u}_{j}^{n+1}-\tilde{u}_{j}^{n}\right|^{2} \\
& +\frac{\tau \kappa \mu}{1+\kappa} \sum_{j}\left|M_{j}^{n}\right|^{2}+\frac{\tau \mu^{2}}{2(1+\kappa)} \sum_{j}\left|N_{j}^{n}\right|^{2} \\
& +\frac{\tau \lambda \kappa}{1+\kappa} \sum_{j}\left(\lambda_{k, j}^{n+1}-\lambda_{k, j+1}^{n+1}\right)\left|\tilde{u}_{k, j}^{n+1}\right|^{2}
\end{aligned}
$$


and

$$
\begin{aligned}
& R(n)=\sum_{j}\left|\tilde{P}_{j}^{n+1}-\tilde{P}_{j}^{n}\right|^{2}-\sum_{j} \frac{\mu^{2}}{2(1+\kappa)} \Delta_{0} \tilde{P}_{j}^{n} N_{j}^{n} \\
& -\frac{\lambda \kappa}{1+\kappa} \sum_{j}\left(\tilde{P}_{j+1}^{n}+\tilde{P}_{j}^{n}\right) \Lambda_{j+1}^{n+1}\left[\left(L_{j+1}^{n+1}-L_{j-1}^{n+1}\right) \tilde{P}_{j+1}^{n}-\Delta^{+} L_{j}^{n} \tilde{P}_{j}^{n}\right] \\
& -\frac{\lambda \kappa}{1+\kappa}(1-\mu) \sum_{j}\left(\tilde{P}_{j+1}^{n}+\tilde{P}_{j}^{n}\right) \Lambda_{j+1}^{n+1}\left(L_{j}^{n+1} R_{j}^{n} \tilde{u}_{j}^{n}-L_{j+1}^{n+1} R_{j+1}^{n} \tilde{u}_{j+1}^{n}\right) \\
& -\frac{\lambda \kappa \mu}{2(1+\kappa)} \sum_{j}\left(\tilde{P}_{j+1}^{n}+\tilde{P}_{j}^{n}\right) \Lambda_{j+1}^{n+1}\left(L_{j}^{n+1} R_{j+1}^{n} \tilde{u}_{j+1}^{n}-L_{j+1}^{n+1} R_{j+2}^{n} \tilde{u}_{j+2}^{n}\right) \\
& -\frac{\lambda \kappa \mu}{2(1+\kappa)} \sum_{j}\left(\tilde{P}_{j+1}^{n}+\tilde{P}_{j}^{n}\right) \Lambda_{j+1}^{n+1}\left(L_{j}^{n+1} R_{j-1}^{n} \tilde{u}_{j-1}^{n}-L_{j+1}^{n+1} R_{j}^{n} \tilde{u}_{j}^{n}\right) \\
& +\sum_{j} \frac{2}{1+\kappa} \tilde{P}_{j}^{n} \Delta^{+} L_{j}^{n} R_{j}^{n} \tilde{P}_{j}^{n}+\frac{\lambda \kappa}{1+\kappa} \sum_{j} \tilde{P}_{j}^{n} \Lambda_{j}^{n+1} \Delta_{0} L_{j}^{n+1} R_{j}^{n+1} \tilde{u}_{j}^{n+1} \\
& +2 \tau \sum_{j} \Delta^{+} \tilde{u}_{j}^{n} \tilde{P}_{j}^{n}+2 \sum_{j} \frac{\tau \kappa}{1+\kappa} \tilde{u}_{j}^{n+1} \Delta^{+} L_{j}^{n} R_{j}^{n+1} \tilde{u}_{j}^{n+1} \\
& +\frac{\tau \kappa \mu}{1+\kappa} \sum_{j} \Delta^{+} \tilde{u}_{j}^{n} L_{j}^{n}\left(R_{j+1}^{n} \tilde{u}_{j+1}^{n}-2 R_{j}^{n} \tilde{u}_{j}^{n}+R_{j-1}^{n} \tilde{u}_{j-1}^{n}\right) \\
& +\frac{\tau \kappa \mu}{1+\kappa} \sum_{j} \tilde{u}_{j}^{n} L_{j}^{n} \Delta_{+} R_{j}^{n} \Delta_{+} \tilde{u}_{j}^{n}+\frac{\tau \kappa \mu}{1+\kappa} \sum_{j} \tilde{u}_{j}^{n} L_{j}^{n} \Delta_{-} R_{j}^{n} \Delta_{-} \tilde{u}_{j}^{n} \\
& +\frac{\tau \kappa \mu}{1+\kappa} \sum_{j} \tilde{u}_{j}^{n} L_{j}^{n} \Delta R_{j}^{n} \tilde{u}_{j}^{n}+\frac{\tau \mu^{2}}{2(1+\kappa)} \sum_{j} \Delta^{+} \tilde{u}_{j}^{n} \Delta_{0} N_{j}^{n} \\
& +\frac{\tau \mu}{1+\kappa} \sum_{j} \Delta^{+} \tilde{u}_{j}^{n} \Delta P_{j}^{n}-\frac{\tau \mu}{1+\kappa} \sum_{j} \Delta_{+} \tilde{u}_{j}^{n} \Delta_{+} \tilde{P}_{j}^{n} \\
& +\frac{2 \tau}{1+\kappa} \sum_{j} \tilde{u}_{j}^{n+1} \Delta^{+} L_{j}^{n} R_{j}^{n} \tilde{P}_{j}^{n}+\frac{\tau \lambda \kappa}{1+\kappa} \sum_{j} \tilde{u}_{j}^{n+1} \Lambda_{j}^{n+1} \Delta_{0} L_{j}^{n+1} R_{j}^{n+1} \tilde{u}_{j}^{n+1} \\
& -\frac{\lambda \kappa}{1+\kappa} \sum_{\mu \neq k} \tilde{P}_{\mu, j}\left(\Lambda_{\mu, j}^{n+1}-\Lambda_{\mu, j+1}^{n+1}\right) \tilde{P}_{\mu, j}^{n} \\
& -\frac{\tau \lambda \kappa}{1+\kappa} \sum_{\mu \neq k} \tilde{u}_{\mu, j}^{n+1}\left(\Lambda_{\mu, j}^{n+1}-\Lambda_{\mu, j+1}^{n+1}\right) \tilde{u}_{\mu, j}^{n+1}+\sum_{j} 2\left(\tau \tilde{u}_{j}^{n+1}+\tilde{P}_{j}^{n}\right) e_{j}^{n} .
\end{aligned}
$$

Label the terms in $R(n)$ as $R_{1}, \ldots, R_{23}$. Actually, we only need to handle the terms which have $\tilde{u}_{j}^{n+1}$, among which the most difficult terms are $R_{10}$ and $R_{19}$. We have

$$
\begin{aligned}
L_{j}^{n+1} & -L_{j}^{n}+\frac{\lambda s}{2}\left(L_{j+1}^{n}-L_{j-1}^{n}\right) \\
& =(1+O(\epsilon)) \nabla L\left(\phi_{j}^{n}\right) \times\left(\phi_{j}^{n+1}-\phi_{j}^{n}+\frac{\lambda s}{2}\left(\phi_{j+1}^{n}-\phi_{j-1}^{n}\right)\right)
\end{aligned}
$$


and the identity

$$
\begin{aligned}
L_{j}^{n+1} & -L_{j}^{n}+\frac{\lambda}{2} \Lambda_{j}^{n}\left(L_{j+1}^{n}-L_{j-1}^{n}\right) \\
& =L_{j}^{n+1}-L_{j}^{n}+\frac{\lambda s}{2}\left(L_{j+1}^{n}-L_{j-1}^{n}\right)+\frac{\lambda}{2}\left(\Lambda_{j}^{n}-s\right)\left(L_{j+1}^{n}-L_{j-1}^{n}\right) .
\end{aligned}
$$

According to the discrete shock profile equation (4.1), we have

$$
\frac{\lambda}{2}\left|\psi_{j+1}^{n}-\psi_{j-1}^{n}\right| \leq c\left(\lambda_{k, j}^{n}-\lambda_{k, j+1}^{n}\right) .
$$

It is obvious that

$$
\begin{aligned}
R_{10}+R_{19}= & \frac{2 \tau \kappa}{1+\kappa} \sum_{j} \tilde{u}_{j}^{n+1}\left[L_{j}^{n+2}-L_{j}^{n+1}+\frac{\lambda}{2} \Lambda_{j}^{n+1}\left(L_{j+1}^{n+1}-L_{j-1}^{n+1}\right)\right] R_{j}^{n+1} \tilde{u}_{j}^{n+1} \\
& -\frac{\tau \lambda \kappa}{1+\kappa} \sum_{j} \tilde{u}_{j}^{n+1}\left(L_{j}^{n+2}-2 L_{j}^{n+1}+L_{j}^{n}\right) R_{j}^{n+1} \tilde{u}_{j}^{n+1} .
\end{aligned}
$$

Because

$$
\begin{aligned}
\phi_{j}^{n+2} & -\phi_{j}^{n+1}+\frac{\lambda}{2}\left(F\left(\phi_{j+1}^{n+1}\right)-F\left(\phi_{j-1}^{n+1}\right)\right)-\frac{\lambda}{2} A^{\frac{1}{2}}\left(\phi_{j+1}^{n+1}-2 \phi_{j}^{n+1}+\phi_{j-1}^{n+1}\right) \\
= & \frac{\lambda}{2 \kappa}\left[\psi_{j+1}^{n+1}-\psi_{j-1}^{n+1}-\left(\psi_{j+1}^{n}-\psi_{j-1}^{n}\right)+\frac{\lambda}{2} A\left(\phi_{j+2}^{n}-2 \phi_{j}^{n}+\phi_{j-2}^{n}\right)\right. \\
& \left.-\frac{\lambda}{2} A^{\frac{1}{2}}\left(\psi_{j+2}^{n}-\psi_{j}^{n}-2\left(\psi_{j+1}^{n}-\psi_{j-1}^{n}\right)+\psi_{j}^{n}-\psi_{j-2}^{n}\right)\right]
\end{aligned}
$$

and by Theorem 2.3 we have

$$
\begin{aligned}
\phi_{j}^{n+2}-\phi_{j}^{n+1} & +\frac{\lambda}{2} s\left(\phi_{j+1}^{n+1}-\phi_{j-1}^{n+1}\right) \\
& \leq O(\epsilon)\left(\lambda_{k, j}^{n+1}-\lambda_{k, j+1}^{n+1}\right)+\frac{O(1)}{\kappa}\left(\lambda_{k, j}^{n+1}-\lambda_{k, j+1}^{n+1}\right) .
\end{aligned}
$$

Combining the above inequalities, we obtain

$$
\begin{aligned}
R_{10}+R_{19} \leq & \sum_{j} \frac{\lambda \tau \kappa}{8(1+\kappa)}\left(\lambda_{k, j}^{n+1}-\lambda_{k, j+1}^{n+1}\right)\left|\tilde{u}_{k, j}^{n+1}\right|^{2} \\
& +O(1) \frac{\tau \kappa}{1+\kappa} \sum_{j, \mu \neq k}\left(\lambda_{k, j}^{n+1}-\lambda_{k, j+1}^{n+1}\right)\left|\tilde{u}_{\mu, j}^{n+1}\right|^{2}
\end{aligned}
$$


provided that $\kappa$ is suitably large, which is a reasonable assumption. Set

$$
\bar{M}\left(n_{1}\right)=\sup _{n \leq n_{1}}\left(\sum_{j}\left|\tilde{u}_{j}^{n}\right|^{2}+\sum_{j}\left|\tilde{P}_{j}^{n}\right|^{2}\right)^{\frac{1}{2}}
$$

and assume a priori that $\bar{M}\left(n_{1}\right)$ is suitably small. Then clearly we have

$$
\sup _{(j, n), n \leq n_{1}}\left|\tilde{u}_{j}^{n}\right| \leq \bar{M}\left(n_{1}\right)
$$

Under this prior assumption, the other terms can be estimated as in the stationary case. The details are skipped. Now we reach the following inequality.

$$
\begin{aligned}
E(n+1) & -E(n) \\
& +\frac{\lambda \tau \kappa}{4(1+\kappa)} \sum_{j}\left(\lambda_{k, j}^{n+1}-\lambda_{k, j+1}^{n+1}\right)\left|\tilde{u}_{k, j}^{n+1}\right|^{2} \\
& +\frac{\lambda \kappa}{4(1+\kappa)} \sum_{j}\left(\lambda_{k, j}^{n+1}-\lambda_{k, j+1}^{n+1}\right)\left|\tilde{P}_{k, j}^{n}\right|^{2} \\
& +O(1)\left[\sum_{j}\left|\tilde{P}_{j}^{n}\right|^{2}+\sum_{j}\left|\tilde{P}_{j+1}^{n}-\tilde{P}_{j}^{n}\right|^{2}\right. \\
& \left.+\sum_{j}\left|\tilde{u}_{j}^{n+1}-\tilde{u}_{j}^{n}\right|^{2}+\sum_{j}\left|M_{j}^{n}\right|^{2}+\sum_{j}\left|N_{j}^{n}\right|^{2}\right] \\
\leq & O(1) \sum_{j}\left(\lambda_{k, j}^{n+1}-\lambda_{k, j+1}^{n+1}\right) \sum_{\mu \neq k}\left|\tilde{u}_{\mu, j}^{n+1}\right|^{2} .
\end{aligned}
$$

The waves in the transversal directions are bounded by the last term on the righthand side of the above equation. We should estimate the transversal term. Here we will use the idea of vertical estimate, and the remaining part of this section is the estimate about this term.

It was mentioned in [16] that the estimates on transversal waves are technically quite involved due to the wave interactions and the fact that our shocks are nonstationary. The idea of vertical estimate is to relate a sum along time-like discrete segments to a sum over all the grid points in a strip of the space-time plane.

Suppose $\mu>k$. It is the same with another case. For any fixed index $j_{0}$, we define the $k$-th time-like grid line originated at $\left(j_{0}, 0\right)$ to be

$$
J\left(j_{0}\right)=\left\{\left(n, j_{n}\right) \mid j_{n}=j_{0}+(l-1) p \quad \text { for } \quad(l-1)|q| \leq n<l|q|, l=1,2, \ldots\right\} .
$$

For any integer $n, 0 \leq n \leq N q-1$, because the terms which have $\tilde{P}$ can be estimated easily, we multiply the $\mu$-th equation in (4.2) by $2 \tilde{u}_{\mu, j}^{n+1}$ and sum by parts over $j$ from $-\infty$ to $j_{n}$ and $n$ from 1 to $N q-1$. Under the assumption of the prior 
estimate, we obtain the inequality

$$
\begin{aligned}
\sum_{n=0}^{N q-1} & \sum_{j=-\infty}^{j_{n}} \frac{\kappa}{1+\kappa}\left(\tilde{u}_{\mu, j}^{n+1}\right)^{2}-\sum_{n=0}^{N q-1} \sum_{j=-\infty}^{j_{n}} \frac{\kappa}{1+\kappa}\left(\tilde{u}_{\mu, j}^{n}\right)^{2}+\sum_{n=0}^{N q-1} \frac{\kappa}{1+\kappa} \frac{p}{q}\left(\tilde{u}_{\mu, j_{n}}^{n+1}\right)^{2} \\
& +\sum_{n=0}^{N q-1} \frac{\kappa \lambda}{1+\kappa}\left(\lambda_{\mu, j_{n}}^{n+1}-s\right)\left(\tilde{u}_{\mu, j_{n}}^{n+1}\right)^{2}+\frac{c_{1} \kappa \mu}{1+\kappa} \sum_{n=0}^{N q-1} \sum_{j=-\infty}^{j_{n}}\left(\tilde{u}_{\mu, j}^{n}-\tilde{u}_{\mu, j-1}^{n}\right)^{2} \\
& +\frac{c_{2} \mu^{2}}{2(1+\kappa)} \sum_{n=0}^{N q-1} \sum_{j=-\infty}^{j_{n}}\left|N_{\mu, j}^{n}\right|^{2}+\sum_{n=0}^{N q-1} \sum_{j=-\infty}^{j_{n}} \frac{c_{0} \kappa}{1+\kappa}\left(\tilde{u}_{\mu, j}^{n+1}-\tilde{u}_{\mu, j}^{n}\right)^{2} \\
\leq & -\sum_{n=0}^{N q-1} \sum_{j=-\infty}^{j_{n}} 2 \tilde{u}_{\mu, j}^{n+1}\left(\tilde{P}_{\mu, j}^{n+1}-\tilde{P}_{\mu, j}^{n}\right)+O(1) \sum_{n=0}^{N q-1} \sum_{j=-\infty}^{\infty}\left(\lambda_{k, j}^{n+1}-\lambda_{k, j+1}^{n+1}\right)\left|\tilde{u}_{j}^{n+1}\right|^{2} \\
& +\sum_{n=0}^{N q-1} \frac{\kappa}{1+\kappa}\left(\mu-\lambda_{\mu, j_{n}+1}^{n+1}\right) \tilde{u}_{\mu, j_{n}}^{n+1}\left(\tilde{u}_{\mu, j_{n}+1}^{n+1}-\tilde{u}_{\mu, j_{n}}^{n+1}\right)+O(1) \sum_{n=0}^{N q-1} \sum_{j=-\infty}^{\infty}\left|M_{j}^{n}\right|^{2} \\
& +\sum_{n=0}^{N q-1} \frac{\mu^{2}}{2(1+\kappa)} \tilde{u}_{\mu, j_{n}+1}^{n} N_{\mu, j_{n}}^{n}-\sum_{n=0}^{N q-1} \frac{\mu^{2}}{2(1+\kappa)} \tilde{u}_{\mu, j_{n}}^{n} N_{\mu, j_{n}+1}^{n} \\
& +O(1) \sum_{n=0}^{N q-1} \sum_{j=-\infty}^{\infty}\left(\tilde{P}_{j+1}^{n}-\tilde{P}_{j}^{n}\right)^{2}+\sum_{n=0}^{N q-1} \frac{\mu}{1+\kappa} \tilde{u}_{\mu, j_{n}+1}^{n}\left(\tilde{P}_{\mu, j_{n}+1}^{n}-\tilde{P}_{\mu, j_{n}}^{n}\right) \\
& +O(1) \sum_{n=0}^{N q-1} \sum_{j=-\infty}^{+\infty}\left(\lambda_{k, j}^{n+1}-\lambda_{k, j+1}^{n+1}\right)\left|\tilde{P}_{j}^{n}\right|^{2}+O(1) \sum_{n=0}^{N q-1} \sum_{j=-\infty}^{+\infty}\left|\Delta^{+} u_{j}^{n}\right|^{2}
\end{aligned}
$$

where $c_{0}, c_{1}, c_{2}$ are positive constants. Let

$$
\left(E_{\mu, j}^{n}\right)^{2}=\left|\tilde{P}_{\mu, j}^{n}\right|^{2}+2 \tau \tilde{u}_{\mu, j}^{n} \tilde{P}_{\mu, j}^{n}+\frac{\tau \kappa}{1+\kappa}\left|\tilde{u}_{\mu, j}^{n}\right|^{2}
$$

and $\tau=\frac{\kappa}{4(1+\kappa)}$. We have

$$
\begin{aligned}
& \frac{1}{\tau} \sum_{n=0}^{N q-1} \sum_{j=-\infty}^{j_{n}}\left[\left(E_{\mu, j}^{n+1}\right)^{2}-\left(E_{\mu, j}^{n}\right)^{2}\right]+\frac{3 \kappa}{4(1+\kappa)} \sum_{n=0}^{N q-1} \frac{p}{q}\left|\tilde{u}_{\mu, j_{n}}^{n+1}\right|^{2} \\
& \quad+C_{0} \sum_{n=0}^{N q-1}\left|\tilde{u}_{\mu, j_{n}}^{n+1}\right|^{2}+(\text { the same remaining terms) } \\
& \leq O(1) \sum_{n=0}^{N q-1} \sum_{j=-\infty}^{j_{n}}\left[\left|\tilde{P}_{\mu, j}^{n}\right|^{2}+\left|\tilde{P}_{\mu, j}^{n+1}-\tilde{P}_{\mu, j}^{n}\right|^{2}\right]+(\text { the same remaining terms }),
\end{aligned}
$$

where $C_{0}$ is a positive constant. Hence, we should estimate

$$
\frac{1}{\tau} \sum_{n=0}^{N q-1} \sum_{j=-\infty}^{+\infty}\left[\left(E_{\mu, j}^{n+1}\right)^{2}-\left(E_{\mu, j}^{n}\right)^{2}\right]+\frac{3 \kappa}{4(1+\kappa)} \frac{p}{q} \sum_{n=0}^{N q-1}\left|\tilde{u}_{\mu, j_{n}}^{n+1}\right|^{2} .
$$


Immediately, we have

$$
\begin{aligned}
\frac{1}{\tau} \sum_{n=0}^{N q-1} & \sum_{j=-\infty}^{j_{n}}\left[\left(E_{\mu, j}^{n+1}\right)^{2}-\left(E_{\mu, j}^{n}\right)^{2}\right]+\frac{3 \kappa}{4(1+\kappa)} \frac{p}{q} \sum_{n=0}^{N q-1}\left|\tilde{u}_{\mu, j_{n}}^{n+1}\right|^{2} \\
= & \frac{1}{\tau} \sum_{j=-\infty}^{j_{0}+N p}\left(E_{\mu, j}^{N q}\right)^{2}-\frac{1}{\tau} \sum_{j=-\infty}^{j_{0}-p}\left(E_{\mu, j}^{0}\right)^{2}+\frac{3 \kappa}{4(1+\kappa)} \frac{1}{q} \sum_{n=0}^{N-1}\left(p \sum_{\alpha=1}^{q}\left(\tilde{u}_{\mu, j_{n q}}^{n q+\alpha}\right)^{2}\right) \\
& -\frac{1}{q} \sum_{n=0}^{N-1} q \sum_{\beta=0}^{p-1}\left(E_{\mu, j_{n q}-\beta}^{n q}\right)^{2} \frac{1}{\tau}
\end{aligned}
$$

and

$$
\frac{1}{\tau}\left(E_{\mu, j_{n q}-\beta}^{n q}\right)^{2}>\frac{3 \kappa}{4(1+\kappa)}\left(\tilde{u}_{\mu, j_{n q}-\beta}^{n q}\right)^{2},
$$

so

$$
\begin{aligned}
& \frac{3 \kappa}{4(1+\kappa)} \sum_{n=0}^{N q-1} \frac{p}{q}\left|\tilde{u}_{\mu, j_{n}}^{n+1}\right|^{2}-\frac{1}{q} \sum_{n=0}^{N-1} q \sum_{\beta=0}^{p-1}\left(E_{\mu, j_{n q}-\beta}^{n q}\right)^{2} \frac{1}{\tau} \\
& \leq \frac{3 \kappa}{4(1+\kappa)}\left[\frac{1}{q} \sum_{n=0}^{N-1}\left(p \sum_{\alpha=1}^{q}\left(\tilde{u}_{\mu, j_{n q}}^{n q+\alpha}\right)^{2}-q \sum_{\beta=0}^{p-1}\left(\tilde{u}_{\mu, j_{n q}-\beta}^{n q}\right)^{2}\right)\right] .
\end{aligned}
$$

The last term on the right can be estimated as

$$
\begin{aligned}
& \sum_{n=0}^{N-1}\left(p \sum_{\alpha=1}^{q}\left(\tilde{u}_{\mu, j_{n q}}^{n q+\alpha}\right)^{2}-q \sum_{\beta=0}^{p-1}\left(\tilde{u}_{\mu, j_{n q}-\beta}^{n q}\right)^{2}\right) \\
&=p \sum_{n=0}^{N-1} \sum_{\alpha=1}^{q}\left(\tilde{u}_{\mu, j_{n q}}^{n q+\alpha}-\tilde{u}_{\mu, j_{n q}}^{n q}\right)^{2}+2 p \sum_{n=0}^{N-1} \sum_{\alpha=1}^{q}\left(\tilde{u}_{\mu, j_{n q}}^{n q+\alpha}-\tilde{u}_{\mu, j_{n q}}^{n q}\right) \tilde{u}_{\mu, j_{n q}}^{n q} \\
&-q \sum_{n=0}^{N-1} \sum_{\beta=0}^{p-1}\left(\tilde{u}_{\mu, j_{n q}-\beta}^{n q}-\tilde{u}_{\mu, j_{n q}}^{n q}\right)^{2}-2 q \sum_{n=0}^{N-1} \sum_{\beta=0}^{p-1}\left(\tilde{u}_{\mu, j_{n q}-\beta}^{n q}-\tilde{u}_{\mu, j_{n q}}^{n q}\right) \tilde{u}_{\mu, j_{n q}}^{n q} \\
& \leq \frac{O(1)}{\delta} \sum_{j, n}\left(\tilde{u}_{\mu, j}^{n}-\tilde{u}_{\mu, j+1}^{n}\right)^{2}+\frac{O(1)}{\delta} \sum_{j, n}\left(\tilde{u}_{\mu, j}^{n+1}-\tilde{u}_{\mu, j}^{n}\right)^{2}+\delta \sum_{n=0}^{N-1}\left(\tilde{u}_{\mu, j_{n q}}^{n q}\right)^{2} \\
& \leq \frac{O(1)}{\delta} \sum_{j, n}\left(\tilde{u}_{\mu, j}^{n}-\tilde{u}_{\mu, j+1}^{n}\right)^{2}+\frac{O(1)}{\delta} \sum_{j, n}\left(\tilde{u}_{\mu, j}^{n+1}-\tilde{u}_{\mu, j}^{n}\right)^{2} \\
&+\delta \sum_{n=0}^{N-1} \sum_{\alpha=1}^{q}\left(\tilde{u}_{\mu, j_{n q}}^{n q+\alpha}\right)^{2}+\delta\left|u_{\mu, j_{0}}^{0}\right|^{2} \\
&= \frac{O(1)}{\delta} \sum_{j, n}\left(\tilde{u}_{\mu, j}^{n}-\tilde{u}_{\mu, j+1}^{n}\right)^{2}+\frac{O(1)}{\delta} \sum_{j, n}\left(\tilde{u}_{\mu, j}^{n+1}-\tilde{u}_{\mu, j}^{n}\right)^{2} \\
&+\delta \sum_{n=0}^{N q-1}\left(\tilde{u}_{\mu, j_{n}}^{n+1}\right)^{2}+\delta\left|u_{\mu, j_{0}}^{0}\right|^{2},
\end{aligned}
$$


so choosing suitably small $\delta$, we have

$$
\begin{aligned}
\sum_{n=0}^{N q-1}\left(\tilde{u}_{\mu, j_{n}}^{n+1}\right)^{2} \leq & O(1) E(0) \\
& +O(1) \sum_{n=0}^{N q-1} \sum_{j=-\infty}^{\infty}\left(\lambda_{k, j}^{n+1}-\lambda_{k, j+1}^{n+1}\right)\left|\tilde{u}_{j}^{n+1}\right|^{2} \\
& +O(1) \sum_{n=0}^{N q-1} \sum_{j=-\infty}^{\infty}\left|\tilde{u}_{j+1}^{n}-\tilde{u}_{j}^{n}\right|^{2} \\
& +O(1) \sum_{n=0}^{N q-1} \sum_{j=-\infty}^{\infty}\left|\tilde{u}_{j}^{n+1}-\tilde{u}_{j}^{n}\right|^{2} \\
& +O(1) \sum_{n=0}^{N q-1} \sum_{j=-\infty}^{\infty}\left|\tilde{P}_{j+1}^{n}-\tilde{P}_{j}^{n}\right|^{2} \\
& +O(1) \sum_{n=0}^{N q-1} \tilde{u}_{\mu, j_{n}+1}^{n}\left(\tilde{P}_{\mu, j_{n}+1}^{n}-\tilde{P}_{\mu, j_{n}}^{n}\right) \\
& +O(1) \sum_{n=0}^{N q-1} \sum_{j=-\infty}^{\infty}\left[\left|\tilde{P}_{j}^{n+1}-\tilde{P}_{j}^{n}\right|^{2}+\left|\tilde{P}_{j}^{n}\right|^{2}\right] \\
& +O(1) \sum_{n=0}^{N q-1} \tilde{u}_{\mu, j_{n}+1}^{n} N_{\mu, j_{n}}^{n}-O(1) \sum_{n=0}^{N q-1} \tilde{u}_{\mu, j_{n}}^{n} N_{\mu, j_{n}+1}^{n} \\
& +O(1) \sum_{n=0}^{N q-1} \tilde{u}_{\mu, j_{n}}^{n+1}\left(\tilde{u}_{\mu, j_{n}+1}^{n+1}-\tilde{u}_{\mu, j_{n}}^{n+1}\right) .
\end{aligned}
$$

Apply Cauchy's inequality to the above equation and use previous estimates

$$
\begin{aligned}
& \sum_{n=0}^{N q-1}\left(\tilde{u}_{\mu, j_{n}}^{n+1}\right)^{2} \leq O(1) E(0) \\
& \quad+O(1) \sum_{n=0}^{N q-1} \sum_{j=-\infty}^{\infty}\left(\lambda_{k, j}^{n+1}-\lambda_{k, j+1}^{n+1}\right)\left|\tilde{u}_{j}^{n+1}\right|^{2} \\
& +O(1) \sum_{n=0}^{N q-1} \sum_{j=-\infty}^{\infty}\left|\tilde{u}_{j+1}^{n}-\tilde{u}_{j}^{n}\right|^{2} \\
& +O(1) \sum_{n=0}^{N q-1} \sum_{j=-\infty}^{\infty}\left|\tilde{u}_{j}^{n+1}-\tilde{u}_{j}^{n}\right|^{2} \\
& +O(1) \sum_{n=0}^{N q-1} \sum_{j=-\infty}^{\infty}\left|\tilde{P}_{j+1}^{n}-\tilde{P}_{j}^{n}\right|^{2} \\
& +O(1) \sum_{n=0}^{N q-1} \sum_{j=-\infty}^{\infty}\left|\tilde{P}_{j}^{n}\right|^{2} .
\end{aligned}
$$


Multiply both sides by $\lambda_{k, j_{0}}^{0}-\lambda_{k, j_{0}+1}^{0}$ and sum over $j_{0}$ from $-\infty$ to $\infty$. Consequently one will obtain

$$
\begin{aligned}
& \sum_{j_{0}}\left(\lambda_{k, j_{0}}^{0}-\lambda_{k, j_{0}+1}^{0}\right) \sum_{n=0}^{N q-1}\left(\tilde{u}_{\mu, j_{n}}^{n}\right)^{2} \\
& \leq O(\epsilon) E(0)+O(\epsilon) \sum_{n=0}^{N q-1} \sum_{j=-\infty}^{\infty}\left(\lambda_{k, j}^{n+1}-\lambda_{k, j+1}^{n+1}\right)\left|\tilde{u}_{j}^{n+1}\right|^{2} \\
&+O(\epsilon) \sum_{n=0}^{N q-1} \sum_{j=-\infty}^{\infty}\left|\tilde{u}_{j+1}^{n}-\tilde{u}_{j}^{n}\right|^{2} \\
&+O(\epsilon) \sum_{n=0}^{N q-1} \sum_{j=-\infty}^{\infty}\left|\tilde{u}_{j}^{n+1}-\tilde{u}_{j}^{n}\right|^{2} \\
&+O(\epsilon) \sum_{n=0}^{N q-1} \sum_{j=-\infty}^{\infty}\left|\tilde{P}_{j+1}^{n}-\tilde{P}_{j}^{n}\right|^{2} \\
&+O(\epsilon) \sum_{n=0}^{N q-1} \sum_{j=-\infty}^{\infty}\left|\tilde{P}_{j}^{n}\right|^{2} .
\end{aligned}
$$

According to the properties of the discrete shock profile,

$$
\begin{aligned}
& \sum_{n=0}^{N q-1} \sum_{j}\left(\lambda_{k, j}^{n+1}-\lambda_{k, j+1}^{n+1}\right)\left(\tilde{u}_{\mu, j}^{n+1}\right)^{2} \\
& \quad=\sum_{j_{0}} \sum_{n=0}^{N q-1}\left(\lambda_{k, j_{n}}^{n+1}-\lambda_{k, j_{n}+1}^{n+1}\right)\left(\tilde{u}_{\mu, j_{n}}^{n+1}\right)^{2} \\
& \leq(1+O(\epsilon)) \sum_{j_{0}}\left(\lambda_{k, j_{0}}^{0}-\lambda_{k, j_{0}+1}^{0}\right) \sum_{n=0}^{N q-1}\left(\tilde{u}_{\mu, j_{n}}^{n+1}\right)^{2} .
\end{aligned}
$$

Combining this estimate, we have

$$
\begin{aligned}
& \sum_{j, n}\left(\lambda_{k, j}^{n+1}-\lambda_{k, j+1}^{n+1}\right)\left(\tilde{u}_{\mu, j}^{n+1}\right)^{2} \\
& \leq O(\epsilon) E(0)+O(\epsilon) \sum_{j, n}\left(\lambda_{k, j}^{n+1}-\lambda_{k, j+1}^{n+1}\right)\left|\tilde{u}_{j}^{n+1}\right|^{2} \\
&+O(\epsilon) \sum_{j, n}\left|\tilde{u}_{j+1}^{n}-\tilde{u}_{j}^{n}\right|^{2} \\
&+O(\epsilon) \sum_{j, n}\left|\tilde{u}_{j}^{n+1}-\tilde{u}_{j}^{n}\right|^{2} \\
&+O(\epsilon) \sum_{j, n}\left|\tilde{P}_{j+1}^{n}-\tilde{P}_{j}^{n}\right|^{2} \\
&+O(\epsilon) \sum_{j, n}\left|\tilde{P}_{j}^{n}\right|^{2}
\end{aligned}
$$


Similarly for $\mu<k$; therefore, summing over $\mu$ yields

$$
\begin{aligned}
& \sum_{j, n}\left(\lambda_{k, j}^{n+1}-\lambda_{k, j+1}^{n+1}\right) \sum_{\mu \neq k}\left(\tilde{u}_{\mu, j}^{n+1}\right)^{2} \\
& \leq O(\epsilon) E(0)+O(\epsilon) \sum_{j, n}\left(\lambda_{k, j}^{n+1}-\lambda_{k, j+1}^{n+1}\right)\left|\tilde{u}_{j}^{n+1}\right|^{2} \\
& \quad+O(\epsilon) \sum_{j, n}\left|\tilde{u}_{j+1}^{n}-\tilde{u}_{j}^{n}\right|^{2}+O(\epsilon) \sum_{j, n}\left|\tilde{u}_{j}^{n+1}-\tilde{u}_{j}^{n}\right|^{2} \\
& \quad+O(\epsilon) \sum_{j, n}\left|\tilde{P}_{j+1}^{n}-\tilde{P}_{j}^{n}\right|^{2} \\
& \quad+O(\epsilon) \sum_{j, n}\left|\tilde{P}_{j}^{n}\right|^{2} .
\end{aligned}
$$

It follows that

$$
\begin{aligned}
& \sum_{j, n}\left(\lambda_{k, j}^{n+1}-\lambda_{k, j+1}^{n+1}\right) \sum_{\mu \neq k}\left(\tilde{u}_{\mu, j}^{n+1}\right)^{2} \\
& \leq O(\epsilon) E(0)+O(\epsilon) \sum_{j, n}\left(\lambda_{k, j}^{n+1}-\lambda_{k, j+1}^{n+1}\right)\left|\tilde{u}_{k, j}^{n+1}\right|^{2} \\
& \quad+O(\epsilon) \sum_{j, n}\left|\tilde{u}_{j+1}^{n}-\tilde{u}_{j}^{n}\right|^{2}+O(\epsilon) \sum_{j, n}\left|\tilde{u}_{j}^{n+1}-\tilde{u}_{j}^{n}\right|^{2} \\
& \quad+O(\epsilon) \sum_{j, n}\left|\tilde{P}_{j+1}^{n}-\tilde{P}_{j}^{n}\right|^{2} \\
& \quad+O(\epsilon) \sum_{j, n}\left|\tilde{P}_{j}^{n}\right|^{2} .
\end{aligned}
$$

After this estimate, combining (4.10), we prove the following basic estimate.

Proposition 4.1 (A priori estimate). Let $(\tilde{u}, \tilde{P})$ be the solution of (4.2) for $n_{2} \leq$ $n_{1}$. Then there exist positive constants $C, c_{0}$ independent of $n_{1}$ and $\epsilon$ such that

$$
\begin{aligned}
E\left(n_{2}\right)+ & \sum_{j, n \leq n_{2}}\left(\lambda_{k, j}^{n+1}-\lambda_{k, j+1}^{n+1}\right)\left|\tilde{u}_{k, j}^{n+1}\right|^{2} \\
& +\sum_{j, n \leq n_{2}}\left(\lambda_{k, j}^{n+1}-\lambda_{k, j+1}^{n+1}\right)\left|\tilde{P}_{k, j}^{n}\right|^{2} \\
& +c_{0}\left[\sum_{j, n \leq n_{2}}\left|\tilde{P}_{j}^{n}\right|^{2}+\sum_{j, n \leq n_{2}}\left|\tilde{P}_{j+1}^{n}-\tilde{P}_{j}^{n}\right|^{2}\right. \\
& +\sum_{j, n \leq n_{2}}\left|\tilde{u}_{j}^{n+1}-\tilde{u}_{j}^{n}\right|^{2} \\
& \left.+\sum_{j, n \leq n_{2}}\left|M_{j}^{n}\right|^{2}+\sum_{j, n \leq n_{2}}\left|N_{j}^{n}\right|^{2}\right] \\
\leq C E(0) &
\end{aligned}
$$

for all $n_{2} \leq n_{1}$, provided that $\epsilon, \lambda$ and $\bar{M}\left(n_{1}\right)$ are suitably small and $\kappa$ is suitably large. 
By standard continuity argument, the following proposition exists.

Proposition 4.2. Assume that $\epsilon, \frac{1}{\kappa}$ and $\bar{M}(0)$ are suitably small. Then problem (4.2) has a unique global solution $\left(\tilde{u}_{j}^{n}, \tilde{P}_{j}^{n}\right)$ satisfying

$$
\begin{aligned}
\sup _{n} E(n) & +\sum_{j, n}\left(\lambda_{k, j}^{n+1}-\lambda_{k, j+1}^{n+1}\right)\left|\tilde{u}_{k, j}^{n+1}\right|^{2}+\sum_{j, n}\left|\tilde{P}_{j}^{n}\right|^{2}+\sum_{j, n}\left|\tilde{P}_{j+1}^{n}-\tilde{P}_{j}^{n}\right|^{2} \\
& +\sum_{j, n}\left|\tilde{u}_{j}^{n+1}-\tilde{u}_{j}^{n}\right|^{2}+\sum_{j, n}\left|M_{j}^{n}\right|^{2}+\sum_{j, n}\left|N_{j}^{n}\right|^{2} \leq C \bar{M}(0)^{2}
\end{aligned}
$$

for any $n \geq 0$, where $C$ is a positive constant independent of $n$ and $j$. 1.2 .

With this estimate, as before, we can get the $L^{2}$ estimate of $\bar{v}_{j}^{n}$, which is Theorem

\section{ACKNOWLEDGMENT}

This is part of the author's Ph.D. thesis at the Institute of Mathematics Science of the Chinese University of Hong Kong. He would like to express his deepest gratitude to his advisor, Professor Xin Zhouping, who has guided him through all stages of this research and generously shared many ideas with him.

\section{REFERENCES}

1. M. Bultelle, M. Grassin, D. Serre, Unstable Godunov discrete profiles for steady shock waves. SIAM J. Numer. Anal. 35 (1998), 2272-2292. MR 2000k:35189

2. R. Diperna, Convergence of approximate solutions to conservation laws. Arch. Rational Mech. Anal. 82 (1983), 27-70. MR 84k:35091

3. B. Enquist, S. Osher, One-sided difference approximations for nonlinear conservation laws. Math. Comp. 35 (1981), 321-351. MR 82c:65056

4. H. T. Fan, Existence of discrete shock profiles of a class of monotonicity preserving schemes for conservation laws. Math. Comp. 70 (2001), 1043-1069. MR 2001m:65105

5. J. Goodman, Nonlinear asymptotic stability of viscous shock profiles to conservation laws. Arch. Rational Mech. Anal. 95 (1986), 325-344. MR 88b:35127

6. J. Goodman, Z. P. Xin, Viscous limits for piecewise smooth solutions to systems of conservation laws. Arch. Rational Mech. Anal. 121 (1992), 235-265. MR 93k:35167

7. G. H. Hardy, J. E. Littlewood, G. Polya, Inequalities. Cambridge University Press, London, New York, 1943; 2nd edition, 1952. MR 13:727e

8. G. S. Jiang, S. H. Yu, Discrete shocks for finite difference approximations to scalar conservation laws. SIAM J. Numer. Anal. 35 (1998), 749-772. MR 99a:65099

9. S. Jin, Z. P. Xin, The relaxation schemes for system of conservation laws in arbititary space dimensions. Comm. Pure Appl. Math. 48 (1995), 235-276. MR 96c:65134

10. G. Jennings, Discrete shocks. Comm. Pure Appl. Math. 27 (1974), 25-37. MR 49:3358

11. P. D. Lax, Weak solutions of nonlinear hyperbolic equations and their numerical computation. Comm. Pure Appl. Math. 7 (1954), 159-193. MR 16:524g

12. P. D. Lax, Hyperbolic Systems of Conservation Laws and the Mathematical Theory of Shock Waves. SIAM, Philadelphia, 1973. MR 50:2709

13. H. L. Liu, J. H. Wang, T. Yang, Existence of the discrete travelling waves for a relaxing scheme. Appl. Math. Lett. 10 (1997), 117-122.

14. H. L. Liu, Convergence rates to the discrete travelling wave for relaxation schemes. Math. Comp. 69 (2000), 583-608. MR 2000i:65132

15. H. L. Liu, J. H. Wang, T. Yang, Nonlinear stability and existence of stationary discrete travelling waves for the relaxing schemes. Japan J. Indust. Appl. Math. 16 (1999), 195-224. MR 2000d: 65157

16. J. G. Liu, Z. P. Xin, Nonlinear stability of discrete shocks for system of conservation laws. Arch. Rational Mech. Anal. 123 (1993), 217-256. MR 95c:35166 
17. J. G. Liu, Z. P. Xin, $L^{1}$-stability of stationary discrete shocks. Math. Comp. 60 (1993), 233244. MR 93d:35097

18. T. P. Liu, Hyperbolic conservation laws with relaxation. Commun. Math. Phys. 108 (1987), 153-175. MR 88f:35092

19. T. P. Liu, S. H. Yu, Continuum shock profiles for discrete conservation laws. I: Construction. Comm. Pure Appl. Math. 52 (1999), 85-127. MR 2000b:65154

20. T. P. Liu, S. H. Yu, Continuum shock profiles for discrete conservation laws. II. Stability. Comm. Pure Appl. Math. 52 (1999), 1047-1073. MR 2000f:35095

21. A. Majda, J. Ralston, Discrete shock profiles for system of conservation laws. Comm. Pure Appl. Math. 32 (1979), 445-482. MR 81i:35108

22. D. Michelson, Discrete shocks for difference approximations to system of conservation laws. Adv. in Appl. Math. 4 (1984), 433-469. MR 86f:65159

23. R. D. Richtmyer, K. W. Morton, Difference Methods for Initial Value Problems. 2nd ed., Wiley-Interscience, New York, 1967. MR 36:3515

24. Y. S. Smyrlis, Existence and stability of stationary profiles of the LW scheme. Comm. Pure Appl. Math. 43 (1990), 509-545. MR 91d:65143

25. A. Szepessy, Z. P. Xin, Nonlinear stability of viscous shock waves. Arch. Rational Mech. Anal. 122 (1993), 53-103. MR 93m:35125

26. E. Tadmor, The large time behavior of the scalar genuinely nonlinear Lax-Friedrichs scheme. Math. Comp. 43 (1984), 353-368. MR 86g:65162

Institute of Mathematics Science and Department of Mathematics, Chinese UniverSity OF Hong Kong, Hong Kong

Current address: School of Computer Science and Engineering, University of Electronic Science and Technology of China, Sichuan, China 610054

E-mail address: yem_mei29@hotmail.com 